\title{
Implementing Efficient Graphs in Connection Networks*
}

\author{
Ruben Juarez $^{\dagger} \quad$ Rajnish Kumar ${ }^{\ddagger}$
}

First version: October 23, 2009, This version: November 19, 2010.

Working Paper No. 10-22

\begin{abstract}
We consider the problem of sharing the cost of a network that meets the connection demands of a set of agents. The agents simultaneously choose paths in the network connecting their demand nodes. A mechanism splits the total cost of the network formed among the participants.

We introduce two new properties of implementation. The first property, Pareto Nash Implementation (PNI), requires that the efficient outcome is always implemented in a Nash equilibrium, and that the efficient outcome Pareto dominates any other Nash equilibrium. The average cost mechanism $(A C)$ and other asymmetric variations, are the only rules that meet PNI. These mechanisms are also characterized under Strong Nash Implementation.

The second property, Weakly Pareto Nash Implementation (WPNI), requires that the least inefficient equilibrium Pareto dominate any other equilibrium. The egalitarian mechanism ( $E G)$, a variation of $\mathrm{AC}$ that meets individual rationality, and other asymmetric mechanisms are the only rules that meet WPNI and Individual Rationality.

PNI and WPNI provide the first economic justification of the Price of Stability (PoS), a seemingly natural measure in the computer science literature but not easily embraced in economics. EG minimizes the PoS across all individually rational mechanisms.
\end{abstract}

JEL classification: C70, C72, D71, D85.

Keywords: Cost-sharing, Implementation, Average Cost, Egalitarian Mechanism.

*We would like to thank our advisor Hervé Moulin for his invaluable insights at the various stages of this paper. This paper benefited from the comments of seminar participants at École Polytechnique, Universitat Autónoma de Barcelona, SED conference in Maastricht, Network Economics conference at LSU and SCW conference in Moscow. Critical comments by Gustavo Bergantinos, Anna Bogomolnaia, Francis Bloch, Anirban Kar, Tim Roughgarden, Sudipta Sarangi, Arunava Sen and Katya Sherstyuk have been very helpful.

$\dagger$ University of Hawaii, Department of Economics, 2424 Maile Way, Saunders Hall 542, Honolulu, HI 96822. E-mail: rubenj@hawaii.edu, Ph: (808) 956-7143.

${ }^{\ddagger}$ LSU, Department of Economics, 2138 Patrick F. Taylor Hall, Baton Rouge LA 70803 E-mail: rajnishkumar@lsu.edu, Ph: (225) 578-3805 


\section{Introduction}

\section{$1.1 \quad$ Network cost-sharing}

We consider the problem of sharing the cost of a congestion-free network that meets the connection demands of a set of agents. The agents simultaneously choose paths in the network connecting the demand nodes, and a mechanism splits the total cost of the network formed among the participants. This type of problem arises in many contexts ranging from water distribution systems, road networks, telecommunications services and multicast transmission to large computer networks such as the Internet.

The Shapley mechanism (Sh, [4]), which divides the cost of every edge equally among its users, has become focal in this setup. Even though Shapley looks like a natural mechanism in this setting, there are serious problems associated with it. First, this method may provide wrong incentives to the players and they may end up choosing an inefficient graph in equilibrium. Indeed, consider the network in figure 1 right. The equilibrium under the Shapley mechanism is $\left(s t_{1}, s t_{2}\right)$, which has a total cost equal to 2 , whereas the efficient connection network has a cost equal to $\frac{3}{2}+\epsilon$. Even the best equilibrium can be as costly as $H(k)=1+\frac{1}{2}+\cdots+\frac{1}{k}$ times the cost of the optimal graph, where $k$ is the number of users ([3]). The next issue with the Shapley mechanism is its asymmetry at equilibrium. Even though the mechanism is symmetric, at equilibrium it may charge different amounts to agents who are in exactly symmetric situations before the choice of paths by the agents. To see this problem, consider the symmetric network for two agents with common sources and two sinks depicted in the left panel of figure 1. Here, the Nash equilibria of the Shapley mechanism are $\left(s t_{1}, s t_{1} t_{2}\right)$ or $\left(s t_{2} t_{1}, s t_{2}\right)$. Thus agents pay either $\left(\frac{1}{2}, 1-\epsilon\right)$ or $\left(1-\epsilon, \frac{1}{2}\right)$ depending on the equilibrium. Hence, even though the network is symmetric, agents pay different costs at equilibrium under the Shapley mechanism. This example also points to the multiplicity of equilibria and thus the problem of equilibrium selection. The next major concern with this mechanism is that it is not continuous in the network structure. The mechanism is very discontinuous and hence unstable: the two networks in figure 1 can be arbitrarily close under any measure, whereas the equilibria will be arbitrarily different under the Shapley mechanism. Continuity is also desirable since unavoidable measurement errors in practical life may lead to very unfair outcomes.

Finally, we notice that the amount of information needed for the Shapley mechanism may not be practical in many settings. The Shapley mechanism

needs as input the paths chosen by each agent. This information can be out of reach in many settings. Consider for instance the network of roads in a state, district or country to be financed by the users of the roads. The procurement of information on the exact paths used by the drivers needs the compulsory installment of GPS (global positioning system) in all the vehicles and the data to be stored and updated by a central taxing authority. Because of privacy issues this may not be possible politically (see, for example, [14]). However, a tax based on the number of miles driven can be implemented without raising such privacy 

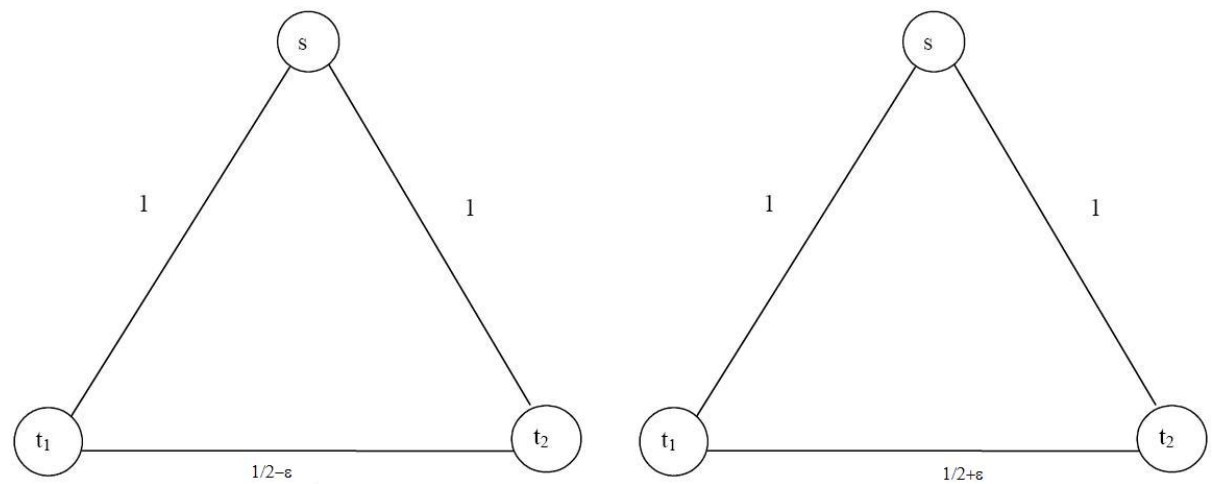

Figure 1: Symmetric networks with a common source and two sinks

concerns. Road maintenance taxes based on the miles driven by every user have been used in pilot programs in Oregon since January 2009, and other states such as Ohio, Pennsylvania, Colorado, Florida, Rhode Island, Minnesota and Texas, are considering them (see $[9,10,11,12,13,14])$. This kind of environment requires mechanisms where the input is the total cost of the paths used by the agents rather than the paths themselves. Moreover, in spite of the information on the paths being available, it may sometimes be desirable to use just the total costs of the paths rather than the paths themselves. Consider, for instance a big or highly dynamic network structure, where agents join and leave the network continuously. It may be impractical to change the formulae of our mechanism every time the network changes. One such example is sharing the cost of a telephone network or the Internet where the agreement is generally monthly but there are agents entering and leaving the network continuously. Notice that charging the same amount for long distance calls makes sense irrespective of the number of users who share the edges. ${ }^{1}$ There are normative concerns too for charging agents who may not be responsible for the fact that their links are not shared by a lot users. Examples are electricity/water supply or postal service to remote villages.

This type of setting demands a new framework that is easy to implement in settings where the inputs of the mechanism are only the total cost of the agents' demands and the total cost of the network formed. This type of problem resembles the classic bankruptcy problem (also referred to in the literature as a rationing or taxation problem), where a given amount of resource (e.g., money) must be divided among beneficiaries with unequal claims on the resource (see [28] [33] for detailed surveys about the problem).

\footnotetext{
${ }^{1}$ The choice of path is not a strategy for the telephone user and thus the setting is not exactly the same. But the cost-sharing method has a similar motivation, namely it is simpler than charging every caller differently based on the path used.
} 


\subsection{Robust efficient implementation}

The celebrated literature on full implementation has more often than not hit impossibilities (see [21] for a comprehensive survey). In the growing literature in computer science (and more recently in economics ${ }^{2}$ ), two measures of efficiency loss have been very fruitfully studied. On the one hand, there is the traditional price of anarchy (PoA [17]), which computes the ratio of the worst equilibrium over the efficient outcome. On the other hand, there is the price of stability (PoS [3]) which computes the ratio of the best equilibrium over the efficient outcome. Both of these measures have been very effective in selecting secondbest mechanisms. However, these approaches lack economic justification and thus seem to be quite arbitrary in the absence of a compelling equilibrium selection rule. Why is a mechanism going to perform in the worst case scenario? Why not the best case scenario?

In this paper, we fill this gap by providing new equilibrium selection rules. We introduce two new properties of implementation. ${ }^{3}$ The first property, Pareto Nash Implementation (PNI), requires that the efficient outcome always be implemented in a Nash equilibrium, and that the efficient outcome Pareto dominates any other Nash equilibrium. Contrary to the traditional literature on full implementation, PNI may implement multiple inefficient equilibria, however the efficient equilibrium is always implemented and Pareto dominates any other equilibrium.

The second property, Weakly Pareto Nash Implementation (WPNI) requires that the least inefficient equilibrium Pareto dominate any other equilibrium. That is, WPNI might implement several equilibria (and all of them might be inefficient), but the least inefficient equilibrium should be preferred by all the agents to any other equilibrium. Thus, if the Nash equilibrium is a good predictor of the outcome implemented by the mechanism, then the least inefficient equilibrium will be implemented since all the agents prefer it.

PNI and WPNI rule out problems of equilibrium selection, since either the efficient equilibrium is implemented under PNI, or the least inefficient is implemented under WPNI. Therefore the price of stability, which uses the best equilibrium as a benchmark is very well justified under PNI and WPNI.

\subsection{Overview of the results}

Theorem 1 characterizes the class of mechanisms that satisfy PNI. The mechanisms are monotonic in the total cost and do not depend on the demands of the agents. The average cost mechanism (AC) ([27] [16]) which divides the to-

\footnotetext{
${ }^{2}$ See for instance [25] for a comparison of three cost-sharing mechanisms using the price of anarchy. See [16] for a comparison of two mechanisms in the problem of commons using the worst-absolute surplus loss.

${ }^{3}$ Notice that the problem of implementation we consider here differs from the one considered in the traditional literature since there is no private information on the part of agents. However, it is the same problem in the sense that the planner has an objective function (here efficiency) and the cost-sharing mechanism induces a game whose equilibrium is the outcome obtained.
} 
tal cost of the network equally among its participants (Theorem 2) is the only symmetric mechanisms in this class. These mechanisms are also characterized under Strong Nash Implementability, which requires the efficient equilibrium to be a Strong Nash.

The main downside of $\mathrm{AC}$ and the above variations is that they do not meet individual rationality (IR, also referred to in the literature as voluntary participation): agents demanding cheap links may pay more than the cost of their demands, thus they may subsidize agents who demand expensive links. We provide a class of mechanisms that meet IR and meet WPNI. The egalitarian mechanisms (EG), a rule reminiscent to AC that meets IR, meets WPNI. Theorem 3 characterizes non-symmetric variations of EG as the only rules that meet WPNI.

EG is no more wasteful than the Shapley mechanism. It has a PoS equal to $H(k)=1+\frac{1}{2}+\cdots+\frac{1}{k}$, where $k$ is the number of agents in the network. EG is also an optimum across all rules meeting IR under the PoS measure. This is remarkable since, as we have discussed above, EG requires much less information than Sh. Finally, the proportional method, a seemingly natural method in this framework, also admits a pure strategy Nash equilibrium but is far more inefficient than the egalitarian rule.

\subsection{Related literature}

The performance of Sh has been widely studied in the recent literature. For example, [4] studies the equilibrium behavior of separable mechanisms, a class of decentralized mechanisms that divides the cost of each edge among its users. The PoS of separable mechanisms with a linear cost-sharing function is at least $H(k)$ (which is $O(\log k)$ ), where $k$ is the number of agents [4]. $H(k)$ is the upper bound on $\mathrm{PoS}(\mathrm{Sh})$ in general graphs, [3]. This upper bound is achieved in directed graphs. If the graph is undirected, $\mathrm{PoS}(\mathrm{Sh})$ is lower than $H(k)$. [1] finds a new upper bound of $O(\log \log k)$ when the graph is single source and there are no steiner nodes. [19] finds a new upper bound of $O(\log k / \log \log k)$ for single source networks when steiner nodes are allowed. [4] shows that the upper bound in two player case with single source is $\frac{4}{3}$. [18] finds out that

$\frac{4}{3}$ is also the upper bound in general multi commodity case. [6] investigates the conditions on network topologies that admit strong equilibrium under $S h$ and finds the upper bound on Strong Price of Anarchy ([2]) under $S h$ to be $H(k)$. [8] considers a similar problem as ours where the designer's objective is to implement the minimum cost spanning tree but the private information about the link costs are not known to the designer. They characterize the set of cost-sharing rules under which true revelation of link costs is a NE.

\section{The Model}

We fix the number of agents $\bar{K}=\{1,2, \ldots, k\}$. A network cost-sharing problem is a tuple $N=\langle G, K>$, where $G=(V, E)$ is a network that is directed 
or undirected such that each edge $e \in E$ has a non-negative $\operatorname{cost} c_{e}$. $K=$ $\left\{\left\{s_{1}, t_{1}\right\},\left\{s_{2}, t_{2}\right\}, \ldots,\left\{s_{k}, t_{k}\right\}\right\}$, where $\left\{s_{i}, t_{i}\right\} \in 2^{V}$ for all $i \in \bar{K}$, is the set of sources and sinks that agents want to connect. When there is no confusion, we also denote $K=\bar{K}$ the set of agents. Let the set of all graphs be $\mathbf{G}$, and the set of all network cost-sharing problems be denoted by $\mathbf{N}$.

Given a problem $N \in \mathbf{N}$, a strategy for agent $i$ is a path $P_{i} \subseteq E$ that connects $s_{i}$ to $t_{i}$. Let the set of paths connecting $s_{i}$ to $t_{i}$ be $\Pi_{i}(N)$. Let $\Pi(N) \equiv \underset{i \in K}{\times} \Pi_{i}(N)$ be the set of strategy profiles of all agents in network $N . P=\left\{P_{i}\right\}_{i=1}^{k} \in \Pi(N)$ will be used to denote a strategy profile of the agents. When there is no confusion we denote $\Pi_{i}(N)$ and $\Pi(N)$ simply as $\Pi_{i}$ and $\Pi$ respectively. Let $G_{P}=\cup_{i \in \bar{K}} P_{i}$ be the network formed by the choice of paths by different agents. Let $C(P)=$ $\sum_{e \in G_{P}} c_{e}$ be the cost of the graph formed by strategies $P$.

Let $\mathcal{N}=\cup_{N \in \mathbb{N}} P(N) \times N$ the union of all problems with their respective strategies.

Definition 1 A cost-sharing mechanism is a mapping $\varphi: \mathcal{N} \rightarrow \mathbb{R}_{+}^{k}$ such that

$$
\sum_{i \in K} \varphi_{i}(P, N)=C(P) \text { for all }(P, N) \in \mathcal{N} \text {. }
$$

A cost-sharing mechanism assigns non-negative cost-shares to the users of the network based on their demands such that the total cost of the network formed is exactly collected.

Example 1 - The Shapley mechanism, Sh, divides the cost of every link equally across it users, that is, $S h_{i}(P, N)=\sum_{e \in P_{i}} \frac{c_{e}}{U(e, P)}$ for all $i \in \bar{K}$, where $U(e, P)$ is the number of users of link $e$ in the strategy profile $P$.

- The proportional to stand-alone mechanism, $\eta^{p r}$, divides the cost of the network in proportion to every user's stand-alone cost. That is, $\eta_{i}^{p r}(P, N)=$ $\frac{S A_{i}(N)}{S A_{1}(N)+\cdots+S A_{k}(N)} C(P)$ for all $i \in \bar{K}$, where $S A_{i}(N)=\min _{P_{i} \in \Pi(N)} C\left(P_{i}\right)$ $i s$ the stand-alone of agent $i$ in network $N$.

- The Average Cost mechanism AC divides the cost of the network formed equally across all users. That is $A C_{i}(P, N)=\frac{C(P)}{k}$ for all $i \in \bar{K}$.

The Shapley mechanism is a separable mechanism; that is, it divides the cost of every link only across its users and adds those costs for all links in the network formed. Alternative separable mechanisms can be constructed by considering different cost-sharing rules for the links, for instance, by giving priority across all users. Nevertheless, $S h$ is the optimal mechanism (using the price of stability measure; see below) across all separable mechanism ([4]). Sh can be computed in polynomial time.

On the other hand, $\eta^{p r}$ divides the cost of the network in proportion to the stand-alone of the agents. Since the stand-alone of every agent has to be computed for every network, this mechanism uses the full information of the network. 
$\mathrm{AC}$ divides the cost of the network formed equally across the users of the network. It is the most egalitarian rule, reminiscent of the classic head tax rule where the size of agents' demands is not relevant, only the size of the total cost of the network formed. AC uses less information than $S h$ or $\eta^{p r}$, since only the total cost of the network formed and the number of agents is needed to compute the cost-sharing allocation. There is no need to know the standalone of the agents, or the users of certain links. As a result, its computation complexity is minimal.

Definition 2 A cost-sharing mechanism $\varphi$ is network independent if for any two problems $N=<G, K>$ and $N^{\prime}=<G^{\prime}, K^{\prime}>$ and strategies $P \in P(N)$ and $P^{\prime} \in P\left(N^{\prime}\right)$ such that $C\left(P_{i}\right)=C\left(P_{i}^{\prime}\right)$ for all $i \in \bar{K}$ and $C(P)=C\left(P^{\prime}\right)$ : $\varphi(P, N)=\varphi\left(P^{\prime}, N^{\prime}\right)$.

Network independence captures those mechanisms that depend only on the cost of the network formed and the cost of the agents' demands. Neither Sh nor $\eta^{p r}$ are network independent. On the other hand, AC uses only the total cost of the network formed and the number of users, thus it is network independent. More complex network independent mechanisms are discussed below.

Let $\mathcal{S}^{k}=\left\{(c ; y) \in \mathbb{R}_{+} \times \mathbb{R}_{+}^{k} \mid \max _{i} y_{i} \leq c \leq \sum_{i} y_{i}\right\}$.

Lemma 1 A cost-sharing mechanism $\varphi$ is network independent if and only if there is a unique function $\xi: \mathcal{S}^{k} \rightarrow \mathbb{R}_{+}^{k}$ such that $\sum_{i} \xi_{i}(c ; y)=c$ for all $(c ; y) \in$ $\mathcal{S}^{k}$, and

$$
\varphi(P, N)=\xi\left(C(P) ; C\left(P_{1}\right), \ldots, C\left(P_{k}\right)\right)
$$

for all problems $(P, N) \in \mathcal{N}$.

Proof. The sufficiency part is obvious. We prove the necessity only.

First, for any $(c ; y) \in \mathcal{S}^{k}$ we construct the network $\tilde{N}(c ; y)$ as follows. Assume without loss of generality that $y_{1} \geq y_{2} \geq \cdots \geq y_{k}$. Choose $i, i \in\{1, \ldots k\}$ such that:

$$
y_{1}+y_{2}+\cdots+y_{i} \leq c<y_{1}+y_{2}+\cdots+y_{i+1} .
$$

Let $\tilde{N}(c ; y)$ be a linear network such that every agent has a unique strategy (see figure 2). All agents 1 to $i$ have demand $y_{i}$ that do not intersect. Agent $i+1$ has demand $y_{i+1}$ such that a segment of length $c-\left(y_{1}+y_{2}+\cdots+y_{i}\right)$ does not intersect the other agents, and $y_{1}+y_{2}+\cdots+y_{i+1}-c$ intersects the demand of agent $i$. Agent $j, j>i+1$ has demand $\tilde{y}_{j}$ contained in the demand of the agent 1 .

Clearly, the unique strategy of agent $k$ in $\tilde{N}(c ; y)$ is $y_{k}$, and the network formed by all strategies has cost $c$. Define $\xi: \mathcal{S}^{k} \rightarrow \mathbb{R}_{+}^{k}$ as $\xi(c ; y)=\varphi(\tilde{N}(c ; y))$.

Second, consider any arbitrary network $N=<G, K>$ and a set of demands $P$. On the one hand, notice that $C(P) \geq C\left(P_{i}\right)$ for every agent $i$, since $P_{i} \subseteq P$. On the other hand, notice that $C(P) \leq C\left(P_{1}\right)+\cdots+C\left(P_{k}\right)$, since $P \subseteq P_{1} \cup$ $P_{2} \cup \cdots \cup P_{k}$. 


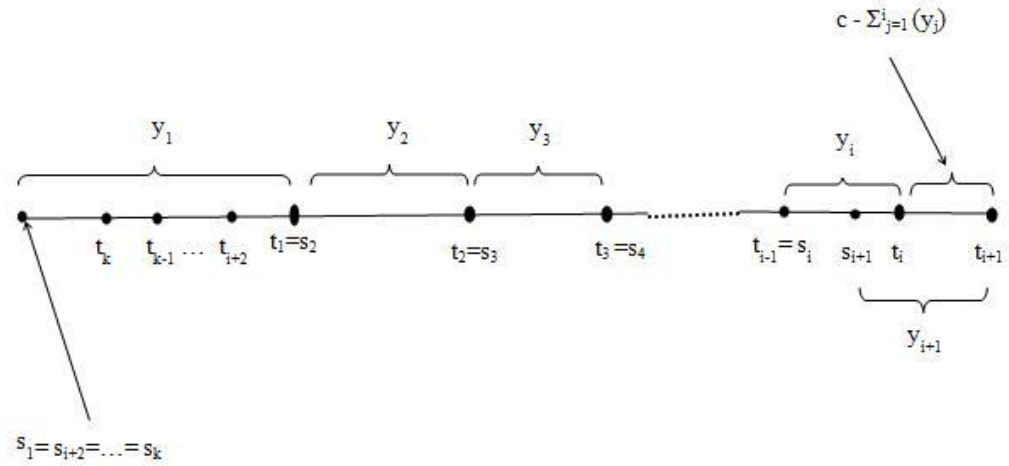

Figure 2: Linear network with all the agents going in the same direction.

Let $y_{i}=C\left(P_{i}\right)$ and $c=C(P)$. Then $(c ; y) \in \mathcal{S}^{k}$. By network independence: $\varphi(P, N)=\varphi(\tilde{N}(c ; y))=\xi(c ; y)$. The uniqueness of $\xi$ follows because it is well defined on $\mathcal{S}^{k}$.

Notice that a network-independent mechanism is reduced to the function $\xi$ that is similar to a taxation (rationing, bankruptcy) solution $([33,28])$. Since we work only on mechanisms that are network independent, we refer without loss of generality to the function $\xi$ as a mechanism. When there is no confusion, the total cost of a path demanded by an agent will be referred to as his demand. We describe below some desirable properties of the function $\xi$.

Definition 3 A mechanism is continuous if the function $\xi: \mathcal{S}^{k} \rightarrow \mathbb{R}_{+}^{k}$ is a continuous function with the Euclidean distance.

Continuous mechanisms capture the fact that small perturbations on the demand or cost of the network should not change the total allocation of the cost. All the network-independent mechanisms described in this paper meet continuity. Continuity is used in all the results without referring to it.

Given a problem $N=<G, K>$, we say $P^{*}$ is an efficient graph if $P^{*} \in$ $\arg \min C(P)$. That is, $P^{*}$ is a graph that connects all the agents at a minimal $P \in \Pi(N)$

cost. Let $\operatorname{Eff}(N)$ be the set of efficient graphs in the network $N$.

Given the problem $N=<G, K>$, the mechanism $\xi$ induces the following non-cooperative game $\Gamma^{\xi}(N) \equiv<\bar{K},\left\{\Pi_{i}(N)\right\}_{i \in \bar{K}},\left\{\xi_{i}\right\}_{i \in \bar{K}}>$, where the representation of the game is the standard representation of the game in normal form. Namely, $\bar{K}=\{1, \ldots, k\}$ is the set of players, $\Pi_{i}(N)$ is the strategy space of player $i$, and $\xi_{i}$ is the (negative of) payoff function of player $i$ that maps a strategy profile to real numbers.

$P$ is a Nash Equilibrium $(N E)$ of $\Gamma^{\xi}(N)$, if $P_{i} \in \arg \min \xi_{i}\left(\dot{P}_{i}, P_{-i}\right)$ for all $i$. $\dot{P}_{i} \in \Pi_{i}(N)$ 
Let

$$
N E\left(\Gamma^{\xi}(N)\right) \equiv\left\{P \in \Pi(N) \mid P \text { is a Nash Equilibrium of } \Gamma^{\xi}(N)\right\}
$$

be the set of Nash equilibria of the game $\Gamma^{\xi}(N)$.

Since every agent in the game $\Gamma^{\xi}(N)$ has a finite number of strategies, then it has a finite number of equilibria.

We say that $\xi$ (weakly) implements $P$, if $P \in N E\left(\Gamma^{\xi}(N)\right)$.

Definition 4 The mechanism $\xi$ is efficient (EFF) if it implements an efficient graph for any problem $N$, that is $P^{*} \in N E\left(\Gamma^{\xi}(N)\right)$ for some efficient graph $P^{*}$.

The definition of efficiency just requires an efficient graph to be selected as a Nash equilibrium. This does not preclude other equilibria from being selected.

Definition 5 The price of stability of the mechanism $\xi$ equals:

$$
\max _{N \in \mathcal{N}, P^{*} \in E f f(N)}\left\{\frac{\min _{P \in N E\left(\Gamma^{\xi}(N)\right)} C(P)}{C\left(P^{*}\right)}\right\}
$$

A mechanism is efficient if it has a price of stability equal to one.

Notice that AC is efficient, therefore it has a price of stability equal to one. Indeed, at any efficient strategy profile $P^{*}$, every agent is paying $\frac{C\left(P^{*}\right)}{k}$. If an agent $i$ deviates from $P^{*}$ to $\tilde{P}_{i}$, then he will pay $\frac{C\left(P_{i}, P_{-i}^{*}\right)}{k}$. Clearly, $\frac{C\left(P_{i}, P_{-i}^{*}\right)}{k} \geq$ $\frac{C\left(P^{*}\right)}{k}$ by the optimality of $P^{*}$.

Section 4 discusses a variety of inefficient mechanisms, i.e., with a price of stability greater than one.

Definition 6 The mechanism $\xi$ Pareto Nash Implements (PNI) an efficient graph if for any problem $N$, it implements an efficient graph and that graph Pareto dominates any other equilibrium. That is, for any problem $N$ :

- There is an efficient graph $P^{*}$ such that $P^{*} \in N E\left(\Gamma^{\xi}(N)\right)$, and

- For any other $P \in N E\left(\Gamma^{\xi}(N)\right): \xi\left(P^{*}\right) \leq \xi(P)$.

PNI is a very robust property that guarantees that the efficient allocation is selected even when multiplicity of equilibria arise. In the case of a multiplicity of equilibria, PNI guarantees that all agents would prefer the efficient graph to any other equilibrium. Hence, a multiplicity of equilibria is not an issue.

In particular, this guarantees that whenever there is a multiplicity of equilibria such that agent $i$ prefers equilibrium $P^{i}$ to $P^{j}$, and agent $j$ prefers equilibrium $P^{j}$ to $P^{i}$, there should exist another equilibrium $P^{*}$ (the efficient equilibrium) such that agent $i$ prefers equilibrium $P^{*}$ to $P^{i}$ and agent $j$ also prefers equilibrium $P^{*}$ to $P^{j}$.

The AC mechanism is also PNI. Indeed, at the efficient graph $P^{*}$, this equilibrium would Pareto dominate any other equilibrium $\tilde{P}$ since $\frac{C\left(P^{*}\right)}{k} \leq \frac{C(\tilde{P})}{k}$.

Another point in favor of AC (and its asymmetric variations discussed below) is that it generates an ordinal potential game on the set of players where 
the potential function is equal to the total cost. Since the vast family of decentralized learning/tatonnement mechanisms converge to a Nash equilibrium in a potential game, ${ }^{4}$ then so will in the AC mechanism. Moreover, with the presence of a non-binding coordinator (who knows the optimal path in advance), the agents can easily converge to the BEST Nash equilibrium.

Definition 7 The mechanism $\xi$ Strongly Nash Implements (SNI) an efficient graph if for any problem $N$ it implements an efficient graph in strong Nash equilibrium. That is, for any problem $N$,

- There is an efficient graph $P^{*}$ such that $P^{*} \in N E\left(\Gamma^{\xi}(N)\right)$, and

- For any group of agents $S \subset\{1, \ldots, k\}$, and $P \in \Pi(N)$ such that $P_{-S}=$ $P_{-S}^{*}$, if $\xi_{i}(P)<\xi_{i}\left(P^{*}\right)$ for some $i \in S$, then $\xi_{j}(P)>\xi_{j}\left(P^{*}\right)$ for some $j \in S$.

Under SNI there is no group of agents that can coordinate paths and weakly improve all of them, and at least one agent in the group strictly improve. In particular, this is similar to the strong Nash equilibrium and to the literature on group strategyproofness $([15,24])$.

The AC mechanism is also SNI. Indeed, at any deviation $\tilde{P}_{S}$ of the group of agent $S$ from the efficient graph $P^{*}$, it should be that $\frac{C\left(P^{*}\right)}{k} \leq \frac{C\left(\tilde{P}_{S}, P_{N \backslash S}^{*}\right)}{k}$ for all $i \in S$. Hence no agent in $S$ would strictly improve by deviating.

Definition 8 - The mechanism is demand monotonic (DM) if for all feasible problems $(c ; y),(c ; \tilde{y}) \in \mathcal{S}^{k}$ such that $y_{-i}=\tilde{y}_{-i}$ and $y_{i}<\tilde{y}_{i}$ : $\xi_{i}(c ; y) \leq \xi_{i}(c ; \tilde{y})$.

- The mechanism is strongly demand monotonic (SDM) if for all feasible problems $(c ; y),(c ; \tilde{y}) \in \mathcal{S}^{k}$ such that $y_{-i}=\tilde{y}_{-i}$ and $y_{i}<\tilde{y}_{i}: \xi_{-i}(c ; y) \geq$ $\xi_{-i}(c ; \tilde{y})$.

Demand monotonicity is a weak property that requires that whenever the demand of an agent increases, everything else fixed, his payment should not decrease. Notice that does not preclude the possibility that the payment of other agents would change. Under SDM, the increase in the demand of one agent does not increase the payment of other agents. In particular, notice that SDM implies DM since all of the agents' payments have to add up to a constant.

$\mathrm{AC}$ is clearly strongly demand monotonic since $A C(c ; y)=A C(c ; \tilde{y})$. Thus the increase of the demand of one agent does not change the payments of the other agents.

\footnotetext{
${ }^{4}$ See $[22,23]$ for convergence of fictitious play and best reply dynamics. See [31] for more general dynamics.
} 


\section{Implementing the Efficient Equilibrium}

We now turn to the first main result of the paper. We characterize the mechanisms that meet the efficiency properties discussed above.

Theorem 1 Assume there are three or more agents, then the following statements are equivalent for the mechanism $\xi$ :

1. $\xi$ is EFF and SDM.

2. $\xi P N I$ the efficient graph.

3. $\xi$ SNI the efficient graph.

4. There is a monotonic function $f: \mathbb{R}_{+} \rightarrow \mathbb{R}_{+}^{k}$ such that $\sum_{i} f_{i}(c)=c$ and for all feasible problems $(c ; y), \xi(c ; y)=f(c)$.

The mechanisms characterized by theorem 1 are demand independent; that is, the cost-share of every agent does not depend on whether the agents are demanding cheap or expensive links. Instead, they depend only on the total cost of the network formed. The average cost mechanism, generated by $f(c)=$ $\left(\frac{c}{k}, \ldots, \frac{c}{k}\right)$, is the only mechanism in this class that treats equal agents equally.

Notice efficiency alone is not sufficient to characterize the above mechanisms. Indeed, consider the mechanism

$$
\tilde{\xi}(c ; y)=\left(\min \left\{y_{3}, \frac{c}{k}\right\}, \frac{2 c}{k}-\min \left\{y_{3}, \frac{c}{k}\right\}, \frac{c}{k}, \ldots, \frac{c}{k}\right) .
$$

First, notice that $\tilde{\xi}$ implements the efficient graph because at the efficient graph agents $\{3, \ldots, k\}$ do not have the incentive to deviate since by doing so their payment is going to increase. On the other hand, agents $\{1,2\}$ do not have any incentive to deviate from the efficient equilibrium since the functions $\min \left\{y_{3}, \frac{c}{k}\right\}$ and $\frac{2 c}{k}-\min \left\{y_{3}, \frac{c}{k}\right\}$ are weakly monotonic in the total cost of the network and do not depend on their report.

$\tilde{\xi}$ is also an example of a mechanism that is not SNI, but agents cannot strictly improve by coordinating.

\subsection{Efficient mechanisms for two agents}

The example above shows that for three or more agents, EFF is not enough to characterize the demand-independent rules. On the other hand, this property is enough when there are two agents. The property is an immediate consequence of a separability lemma described below.

Proposition 1 Assume there are two agents, $K=\{1,2\}$. A mechanism is efficient if and only if there is a monotonic function $f: \mathbb{R}_{+} \rightarrow \mathbb{R}_{+}^{2}$ such that $f_{1}(c)+f_{2}(c)=c$ and for all feasible problems $(c ; y), \xi(c ; y)=f(c)$. 


\subsection{Equal treatment of equals}

Definition 9 The mechanism satisfies equal treatment of equals (ETE) if for all agents $i, j$ and $(c ; y) \in \mathcal{N}^{k}$ such that $y_{i}=y_{j}: \xi_{i}(c ; y)=\xi_{j}(c ; y)$.

ETE is the standard property of equal responsibility for the cost of the good. Equal agents with the same demand should be allocated the same cost. There is a large class of solutions that meet ETE. We describe in section 4 alternative rules that meet ETE, such as the Proportional and Egalitarian solution.

Theorem 2 A mechanism is EFF and ETE if and only if it is $A C$.

Notice that this statement is not directly implied by Theorem 1 , since we do not need SDM.

Alternatively, Theorem 2 implies that AC is the only ETE mechanism with a PoS equal to one.

\section{Individually Rational Mechanisms}

Definition 10 A mechanism $\xi$ is individually rational (IR) if for all $(c ; y) \in$ $\mathcal{S}^{k}: \xi_{i}(c ; y) \leq y_{i}$ for all $i$.

Individually rational mechanisms rule out cross-subsidies; that is, no agent pays more than the cost of their demand.

Notice that neither $A C$ nor any mechanism discussed in Theorem 1 meet individual rationality. Therefore, a reminiscent incompatibility of strategyproofness, efficiency, budget-balance and individual rationality ([7]) also holds in this problem.

This incompatibility holds only because we consider network-independent mechanisms. If we remove network-independence, there is a large class of mechanisms that always implement the efficient network and at the same time meet individual rationality. For instance, consider the proportional to stand-alone mechanism $\eta^{p r}$ discussed above. $\eta^{p r}$ is individually rational because no agent pays more than his stand-alone, which in turn is less than his demand. On the other hand, $\eta^{p r}$ implements the efficient allocation because the cost-share of every agent is in proportion to the cost of the network; therefore, any deviation from the efficient graph that increases the total cost of the network formed would increase the cost-share of all the agents.

On the other hand, there is a large class of individually rational mechanisms that are network independent: most of the mechanisms discussed in the rationing/bankruptcy literature meet IR; see, for instance, [33, 28].

A class of rationing mechanisms that is especially compelling is the class of asymmetric parametric methods.

Definition 11 For every agent $i$, consider $F_{i}:[0, \Lambda] \times \mathbb{R}_{+} \rightarrow \mathbb{R}_{+}$, continuous in both variables, non-decreasing in the first variable and such that $F_{i}(\lambda, 0)=0$ 
and $F_{i}(\Lambda, z)=z$ for all $\lambda$ and $z$. A parametric rationing mechanism is defined as

$$
\varphi_{i}(c, y)=F_{i}\left(\lambda, y_{i}\right) \text { where } \lambda \text { solves } \sum_{i \in \bar{K}} F_{i}\left(\lambda, y_{i}\right)=c .
$$

The class of asymmetric parametric methods is very rich; it contains almost any rationing method discussed in the literature. ${ }^{5}$ In particular, it contains the two basic rationing methods: the proportional and egalitarian mechanisms. The proportional mechanism, $P R$, divides the cost of the agents in proportion to their demands:

$$
P R_{i}(c ; y)=\frac{y_{i}}{y_{1}+\cdots+y_{k}} c .
$$

On the other hand, the egalitarian mechanism, $E G$, divides the cost equally across the agents subject to no agent paying more than their demand:

$$
E G_{i}(c ; y)=\min \left\{y_{i}, \lambda\right\} \text { where } \lambda \text { solves } \sum_{i} \min \left\{y_{i}, \lambda\right\}=c .
$$

The parametric description of these two methods is given by:

Proportional: $F_{i}(\lambda, \mathrm{z})=\lambda z, \Lambda=1$;

Egalitarian: $F_{i}(\lambda, z)=\min \{\lambda, z\}, \Lambda=\infty$;

We now introduce a class of mechanisms that generalize the egalitarian mechanism. These mechanisms, which resembles a fixed path method, are briefly introduced and discussed in section 1.8 of [28]. To illustrate the class of rules, consider a non-decreasing function $f_{i}:[0, \Lambda] \rightarrow \mathbb{R}_{+}$such that $f_{i}(0)=0$ and $f_{i}(\Lambda)=\infty$, for every agent $i \in\{1, \ldots, k\}$. Given the demands of the agents $\left(y_{1}, \ldots, y_{k}\right)$ and a cost of the network $c$, the cost-share of agent $i$ is given by:

$$
E G_{i}^{f_{1}, f_{2}, \ldots, f_{k}}\left(c ; y_{1}, y_{2}, \ldots, y_{k}\right)=\min \left\{f_{i}(\lambda), y_{i}\right\},
$$

where $\lambda$ solves $\sum_{i=1}^{k} \min \left\{f_{i}(\lambda), y_{i}\right\}=c$.

Notice the mechanism $E G_{i}^{f_{1}, f_{2}, \ldots, f_{k}}$ clearly meets IR since

$$
E G_{i}^{f_{1}, f_{2}, \ldots, f_{k}}\left(c ; y_{1}, y_{2}, \ldots, y_{k}\right) \leq y_{i} .
$$

The mechanism $E G_{i}^{f_{1}, f_{2}, \ldots, f_{k}}$ will be called an asymmetric egalitarian mechanism (AEM).

The egalitarian mechanism is constructed by picking functions $f_{1}=f_{2}=$ $\cdots=f_{k}$.

On the other hand, the weighted egalitarian methods are constructed when $f_{i}(\lambda)=w_{i} \lambda$ for all $i$, for a given set of constants $w_{1}, \ldots, w_{k}$.

Contrary to the traditional analysis of this problem, the games induced by the asymmetric egalitarian mechanisms are not potential games, see section 10.2

\footnotetext{
${ }^{5}$ The class of symmetric parametric methods is characterized by consistency, continuity and symmetry; see [34] for the characterization and [28] for a more detailed description of the methods.
} 
for an example illustrating that. Therefore, the previous potential techniques used in the analysis of this problem do not work anymore. We do not know whether any mechanism (induced by a rationing method) always has a pure strategy Nash equilibrium. Nevertheless, we show below that AEM and proportional always have pure strategy Nash equilibria and provide algorithms to compute them.

Lemma 2 The proportional and the asymmetric egalitarian mechanisms always have a pure strategy Nash equilibrium.

Proof. We prove the lemma for an ASM mechanism. The proof for the proportional mechanism is written in the appendix.

Consider the asymmetric egalitarian mechanism $\varphi$ generated by the functions $f^{1}, \ldots, f^{k}$. Let $S_{i}$ be the stand-alone of agent $i$, and let $s^{i}=C\left(S^{i}\right)$ be its cost.

We show the best reply tattonment starting from the profile of demands $\left(S^{1}, S^{2}, \ldots, S^{k}\right)$ converges to a Nash equilibrium.

Let $g^{i}(x)=\left(f^{i}\right)^{-1}(x)$ the inverse of $f^{i}$. Asumme without loss of generality that $g^{1}\left(s^{1}\right) \leq g^{2}\left(s^{2}\right) \leq \cdots \leq g^{k}\left(s^{k}\right)$.

Let $C\left(S^{1}, \ldots, S^{k}\right)$, and let $\lambda^{*}$ such that $\sum_{i} \min \left\{f^{i}\left(\lambda^{*}\right), s^{i}\right\}=C\left(S^{1}, \ldots, S^{k}\right)$.

Let $m$ such that:

$$
g^{1}\left(s^{1}\right) \leq \cdots \leq g^{m-1}\left(s^{m-1}\right) \leq \lambda^{*}<g^{m}\left(s^{m}\right) \leq \cdots \leq g^{k}\left(s^{k}\right) .
$$

First, notice that $\varphi_{i}\left(s_{1}, \ldots, s_{k}\right)=s^{i}$ if $i<m$, and $\varphi_{i}\left(s_{1}, \ldots, s_{k}\right)=f^{i}\left(\lambda^{*}\right)$ if $i \geq m$.

Second, notice that at the best reply of any agent, the variable $\lambda^{*}$ should decrease.

To see this, consider agent $j$ and assume that his best reply is the path $Y^{j}$ with cost $C\left(Y^{j}\right)=y^{j}$.

Case 1. If $j<m$, then his cost-share is $\varphi_{j}\left(s_{1}, \ldots, s_{k}\right)=s^{j}$. At his best reply $Y^{j}, y^{j} \geq s^{j}$. The only way to decrease his cost-share is by moving to $\tilde{\lambda}$ such that $f^{j}(\tilde{\lambda})<s^{j}$. Since $g^{j}\left(s^{j}\right) \leq \lambda^{*}$, then $s^{j} \leq f^{j}\left(\lambda^{*}\right)$. Hence $\tilde{\lambda}<\lambda^{*}$.

Case 2. f $j \geq m$ then $\varphi_{j}\left(s_{1}, \ldots, s_{k}\right)=f^{j}\left(\lambda^{*}\right)<s^{j}$. At his best reply $Y^{j}$, $C\left(Y^{j}\right) \geq s^{j}$. Therefore, the only way to decrease his payment is by decreasing $\lambda^{*}$, since he cannot decrease his demand below $s^{j}$.

Finally, notice we can replicate cases 1 and 2 above for the new profile $\left(Y_{j}, S_{-j}\right)$. Indeed, notice $\varphi_{i}\left(y_{j}, s_{-j}\right)=s^{i}$, or $\varphi_{i}\left(y_{j}, s_{-j}\right)=f^{i}(\tilde{\lambda})$. Similar to case 1 , an agent paying his stand-alone, that is $\varphi_{i}\left(y_{j}, s_{-j}\right)=s^{i}$, will be demanding his stand-alone. Thus, his only profitable deviation will be to decrease $\tilde{\lambda}$ to $\bar{\lambda}$, $\bar{\lambda}<\tilde{\lambda}$, such that $f^{i}(\bar{\lambda})<s^{i}$. Thus, decreasing the total cost of the network.

On the other hand, if $\varphi_{i}\left(y_{j}, s_{-j}\right)=f^{i}(\tilde{\lambda})$ then $f^{i}(\tilde{\lambda}) \leq s^{i}$. Similar to case 2 , an increase in their demand is only profitable if $\tilde{\lambda}$ decreases, and so does the total cost of the network.

Since at any step the value $\lambda^{*}$ decreases, it is bounded and there is a finite number of strategies, then $\lambda^{*}$ converges in a finite number of iterations. The limit profile is a Nash equilibrium. 


\subsection{Weak Pareto Nash Implementation}

There is no individually rational mechanisms that meet PNI. Weakly Pareto Nash Implementation (WPNI) requires that the least inefficient equilibrium Pareto dominate any other equilibrium. That is, WPNI might implement several equilibria (and all of them might be inefficient), but the least inefficient equilibrium should be preferred by all the agents to any other equilibrium.

Definition 12 A mechanisms is WPNI if:

- It always has at least one Nash equilibrium

- Among the different equilibriums with cost $c_{1} \leq c_{2} \leq \cdots \leq c_{l}$, the costshare of every agent at equilibrium $c_{1}$ is not larger than than his cost-share at equilibrium $c_{h}$ for $h=2, \ldots, l$. That is, the equilibrium with cost $c_{1}$ Pareto dominates other equilibria.

WPNI serves an an equilibrium selection rule. If the Nash equilibrium is a good predictor of the outcome implemented by the mechanism, then the least inefficient equilibrium will be implemented since all the agents prefer it.

Theorem 3 An asymmetric parametric mechanism meets WPNI if and only if it is an asymmetric egalitarian mechanism.

The price of stability $(\mathrm{PoS})$, which computes the ratio between the best efficient equilibrium and the efficient outcome, is a compelling measure of the inefficiency generated by WPNI mechanisms since the agents' incentives are aligned to pick the BEST Nash equilibrium.

Corollary $4 \quad i$. EG has the smallest price of stability across all asymmetric parametric mechanisms meeting WPNI. It has a price of stability equal to $H(k)=1+\frac{1}{2}+\cdots+\frac{1}{k}$.

ii. EG is an optimum across all individually rational mechanisms.

iii. The price of stability of PR is of order $k$.

Since the Shapley mechanism has a PoS equal to $H(k), E G$ is no more inefficient than the Shapley mechanism. No other individually rational mechanism can be more efficient than $E G$ and Shapley. On the other hand, the traditional proportional mechanism is extremely inefficient; since its price of stability is bounded by $k$, its maximal loss approaches that in the limit.

\subsection{Strong Nash Implementation}

Definition 13 A mechanism strongly Nash implements the network with the minimal cost (SNIMC) if

- It always has at least one Nash equilibrium 
- The equilibrium with a minimal cost is a strong Nash equilibrium.

Proposition 2 The asymmetric egalitarian mechanisms SNIMC.

Notice that together with the WPNI property of AEG, this proposition implies that there is one and only one strong Nash equilibrium under AEG. This comes from the fact that the Nash equilibria are Pareto ranked and thus under any NE other than the cheapest NE, the grand coalition has a profitable deviation. This means that the strong price of anarchy (SPoA) of the EG equals the strong price of stability of EG, and they equal $H(k)$. This earmarks another advantage of EG over Sh, since we know that Sh does not always admit Strong NE $[3,4]$ and therefore, SPoA does not exist for Sh.

We conjecture that the only mechanisms that SNIMC are the asymmetric egalitarian mechanisms.

\section{Conclusions}

This paper provides a new perspective to the problem of cost-sharing in networks. In particular, it provides new concepts of implementation and characterize the classes of mechanisms that meet them. The average cost mechanism and reminiscent variations are characterized using those properties.

It also provides the first economic justification for the price of stability, a seemingly natural measure in the computer science literature but not easily embraced in economics. It illustrates the environments where $\mathrm{PoS}$ is the correct measure to use. The egalitarian mechanism, an optimal mechanism using this measure, is singled out in the class of WPNI mechanisms. Moreover, EG outperforms the Shapley mechanism on several grounds including efficiency, stability, and fairness.

Several questions remain open, including a characterization of the continuous mechanisms, the full characterization of the class of SNIMC mechanisms, and

the introduction of compelling behavioral properties that justify the price of anarchy. 


\section{Proof of Theorems 1 and 2}

\subsection{Preliminary Lemmas}

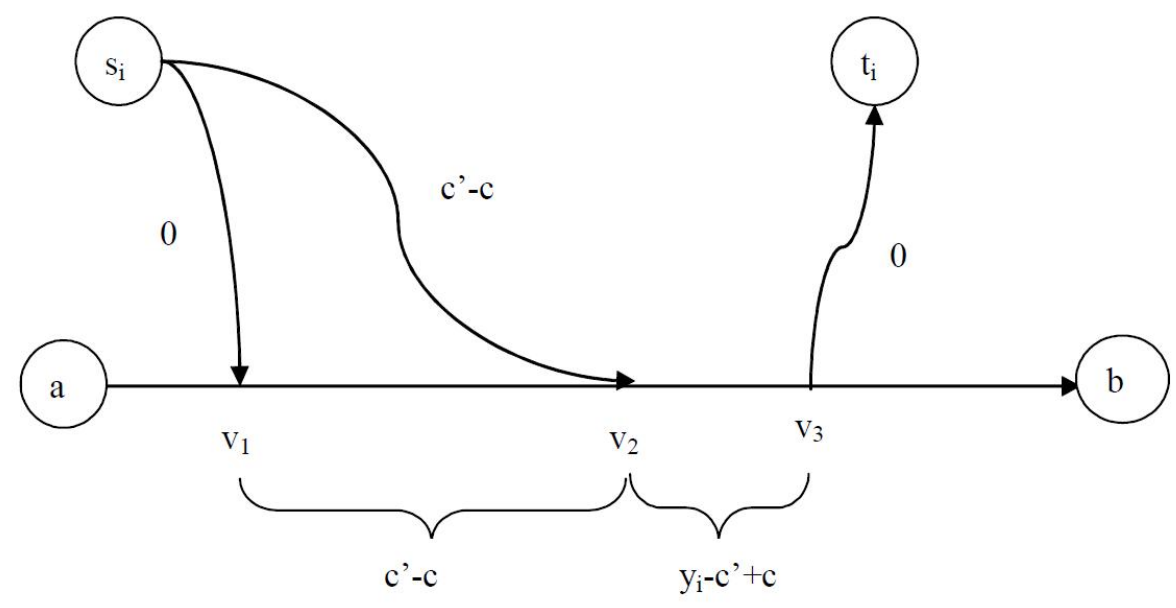

Figure 3: EFF implies cost monotonicity (case 1).

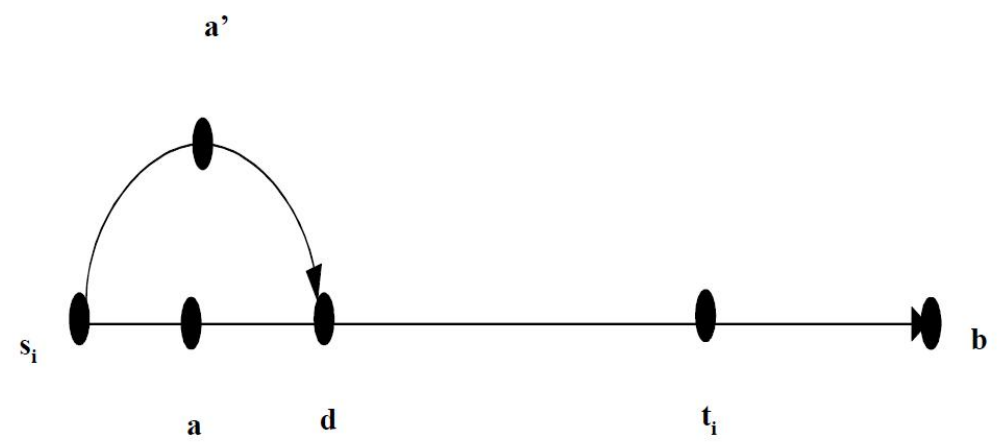

Figure 4: EFF implies cost monotonicity (case 2).

Definition 14 The mechanism is monotonic in cost if for all feasible problems $(c ; y),\left(c^{\prime} ; y\right) \in \mathcal{N}^{K}$ such that $c<c^{\prime}: \xi(c ; y) \leq \xi\left(c^{\prime} ; y\right)$.

Lemma 3 If the mechanism $\xi$ is efficient then it is monotonic in total cost. 


\section{Proof.}

Consider two feasible problems $(c ; y)$ and $\left(c^{\prime} ; y\right)$, where $c^{\prime}>c$ and $\left(c^{\prime}-\right.$ $c)<\min _{i \in K}\left\{y_{i}\right\}$. Suppose there exists an agent $i$ and an efficient $\xi$ such that $\xi_{i}\left(c^{\prime} ; y\right)<\xi_{i}(c ; y)$.

We construct a network that have the two potential profiles $(c ; y)$ and $\left(c^{\prime} ; y\right)$.

Indeed, consider a network where agents $j \neq i$ have just one strategy each, $P_{j}$, which costs $y_{j}$. Agent $i$ has two strategies $P_{i}$ and $P_{i}^{\prime}$ both of which cost $y_{i}$ but $P_{i}$ makes the total cost of the network $c$, and $P_{i}^{\prime}$ makes the total cost go up to $c^{\prime}$.

Case 1: $c \leq \sum_{j \neq i} y_{j}$.

In this case we can have a configuration as shown in figure 3. Here, the demands of agents in $K \backslash\{i\}$ is contained in the interval $a \rightarrow b$, which costs $c$. This is possible since when $c=\sum_{j \neq i} y_{j}$, we can have $a \rightarrow b$ as the concatenation of the demand links of the agents $j \neq i$. When $c<\sum_{j \neq i} y_{j}$, we can have the demand links overlapping, e.g., when $\max _{j \neq i}\left\{y_{j}\right\}=c$, then $a \rightarrow b$ is the demand link of the biggest demander and all other demands overlap with his. $P_{i}=s_{i} \rightarrow v_{1} \rightarrow v_{2} \rightarrow v_{3} \rightarrow t_{i}$ and $P_{i}^{\prime}=s_{i} \rightarrow v_{2} \rightarrow v_{3} \rightarrow t_{i}$. All the costly links of $P_{i}$ are contained in $\left\{\cup_{j \neq i} P_{j}\right\}$ whereas there are links of cost $c^{\prime}-c$ that are not contained in $\left\{\underset{j \neq i}{\cup} P_{j}\right\}$ under $P_{i}^{\prime}$. Again, this is possible since $c^{\prime}$ and $c$ are close enough to guarantee that for all $i$ we can have such paths.

Case $2: \sum_{j \in K} y_{j}>c>\sum_{j \neq i} y_{j}$.

In this case we can have a configuration as shown in figure 4 . Here, the interval $a \rightarrow b$ is the concatenation of the demand links of agents in $K \backslash\{i\}$. Thus $|a \rightarrow b|=\sum_{j \neq i} y_{j},\left|s_{i} \rightarrow a\right|=c-\sum_{j \neq i} y_{j},|a \rightarrow d|=c^{\prime}-c . \quad \mid s_{i} \rightarrow$ $a \rightarrow d|=| s_{i} \rightarrow a^{\prime} \rightarrow d \mid=c^{\prime}-\sum_{j \neq i} y_{j} . \quad P_{i}=s_{i} \rightarrow a \rightarrow d \rightarrow t_{i}$ and $P_{i}^{\prime}=s_{i} \rightarrow a^{\prime} \rightarrow d \rightarrow t_{i}$. Notice that it may be the case that $t_{i}=b$.

Now clearly in both cases, $i$ will have a profitable deviation from the efficient graph of cost $c$, thus contradicting the efficiency of $\xi$. Thus we have shown that efficient $\xi$ must be monotonic in total cost in some open neighborhood of $c$, for all $c$. Therefore, we can extend the argument to conclude that $\xi$ must be monotonic in total cost in general.

Lemma 4 (Separability Lemma) If the mechanism $\xi$ is efficient then $\Longrightarrow$ $\xi(C ; y)=\left(\xi_{1}\left(C ; y_{-1}\right), \xi_{2}\left(C ; y_{-2}\right), \ldots . ., \xi_{k}\left(C ; y_{-k}\right)\right)$. That is, any efficient mechanism is separable and assigns the costs-shares to the agents independently of their demand.

Proof. If we prove that for any feasible problems $(c ; y)$ and $\left(c ; \tilde{y}_{i}, y_{-i}\right)$, any continuous and efficient $\xi$ must have $\xi_{i}(c ; y)=\xi_{i}\left(c ; \tilde{y}_{i}, y_{-i}\right)$, then we are done. Consider a feasible problem $(c ; y)$. Consider a graph as shown in Figure 5 , which generates this problem. The sources and sinks of agents $j \neq i$ lie on the ray $a \rightarrow b$ according to the demand profile; i.e., the agent with the highest demand covers most of the span on $a \rightarrow b$ and so on. Thus, an agent $j \neq i$ has one strategy that generates the demand $y_{j}$. Agent $i$ has two strategies- either connect $s_{i}-t_{i}$ through $v_{1}$ or through $v_{2}$. The demands of agent $i$ when connecting through 


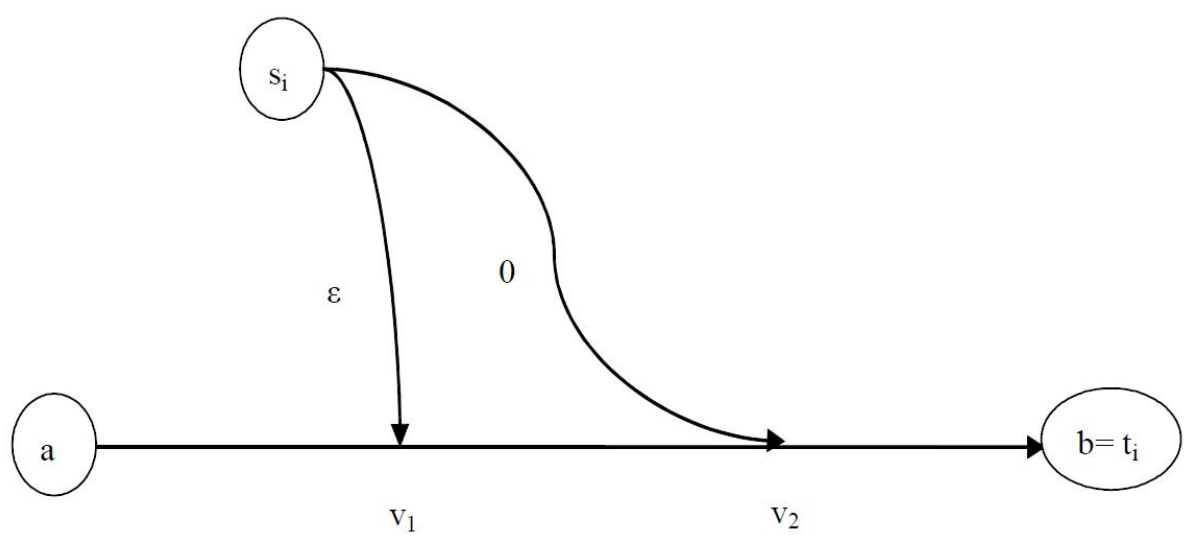

Figure 5: EFF implies separability.

$v_{1}$ and $v_{2}$ are $\tilde{y}_{i}$ and $y_{i}$ respectively. Now, the total cost when $i$ uses $v_{1}$ and $v_{2}$ are respectively $c+\epsilon$ and $c$. Notice that by moving the position of $v_{2}$ and arranging the demand links of the agents $j \neq i$, we can generate all the feasible problems $\left(c ; y_{i}, y_{-i}\right)$. Also, by moving the position of $v_{1}$ and arranging the demand links of the agents $j \neq i$, we can generate all the feasible problems $\left(c+\epsilon ; y_{i}, y_{-i}\right)$. Consider an efficient $\xi$ that is continuous. The efficiency of $\xi$ requires the following inequality

$$
\xi_{i}\left(c ; y_{i}, y_{-i}\right) \leq \xi_{i}\left(c+\epsilon ; \tilde{y}_{i}, y_{-i}\right)
$$

Using continuity we get

$$
\xi_{i}\left(c ; y_{i}, y_{-i}\right) \leq \xi_{i}\left(c ; \tilde{y}_{i}, y_{-i}\right)
$$

Similarly, switching the position of $v_{1}$ and $v_{2}$ and using continuity again we get

$$
\xi_{i}\left(c ; y_{i}, y_{-i}\right) \geq \xi_{i}\left(c ; \tilde{y}_{i}, y_{-i}\right)
$$

Thus, we conclude that $\xi_{i}\left(c ; y_{i}, y_{-i}\right)=\xi_{i}\left(c ; \tilde{y}_{i}, y_{-i}\right)$ for all feasible problems $\left(c ; y_{i}, y_{-i}\right)$ and $\left(c ; \tilde{y}_{i}, y_{-i}\right)$.

\subsection{Proof of Proposition 1}

Consider a problem $\left(c ; y_{1}, y_{2}\right) \in S^{2}$.

By separability lemma: $\xi_{1}\left(c ; y_{1}, y_{2}\right)=\xi_{1}\left(c ; c, y_{2}\right)$.

By budget balance: $\xi_{2}\left(c ; y_{1}, y_{2}\right)=\xi_{2}\left(c ; c, y_{2}\right)$. Thus, $\xi\left(c ; y_{1}, y_{2}\right)=\xi\left(c ; c, y_{2}\right)$.

By separability lemma: $\xi_{2}\left(c ; c, y_{2}\right)=\xi_{2}(c ; c, c)$. 
By budget balance: $\xi_{1}\left(c ; c, y_{2}\right)=\xi_{1}(c ; c, c)$. Thus, $\xi\left(c ; c, y_{2}\right)=\xi(c ; c, c)$.

Therefore $\xi\left(c ; y_{1}, y_{2}\right)=\xi(c ; c, c)$.

Let $f(c)=\xi(c ; c, c)$. Since the mechanism is monotonic in the total cost (lemma 3$), f(c)$ is monotonic in the total cost.

\subsection{Proof of Theorem 1}

\subsection{1 $1 . \Longrightarrow 4$.}

Proof.

Consider a continuous $\xi$ that is efficient and strongly monotonic. Consider two arbitrary feasible problems $(c ; y)$ and $(c ; \tilde{y})$. We will prove that $\xi(c ; y)=$ $\xi(c ; \tilde{y})=f(c)$. The monotonicity of $f$ comes from lemma 1. Let $a=\frac{1}{k} \sum_{i \in K} y_{i}$ and $\tilde{a}=\frac{1}{k} \sum_{i \in K} \tilde{y}_{i}$. Assume without loss of generality that $y_{1} \leq y_{2} \leq y_{3} \leq$ $\ldots \ldots . \leq y_{k}$ and $\tilde{y}_{1} \leq \tilde{y}_{2} \leq \tilde{y}_{3} \leq \ldots \ldots . \leq \tilde{y}_{k}$.

Step 1: $\xi(c ; y)=\xi(c ; a, a, \ldots, a)$ and $\xi(c ; \tilde{y})=\xi(c ; \tilde{a}, \tilde{a}, \ldots ., \tilde{a})$

Proof:

Consider the following problems: $P_{0}=(c ; y), P_{1}=\left(c ; a, y_{2}, y_{3}, \ldots y_{k}\right), P_{2}=$ $\left(c ; a, a, y_{3}, y_{4}, \ldots, y_{k}\right), \ldots \ldots \ldots, P_{k}=(c ; a, a, \ldots \ldots, a)$. Notice first that the feasibility of $P_{0}$ implies the feasibility of $P_{1}, P_{2}, \ldots, P_{k}$. This is true because the maximum of the demand profile does not go above $y_{k}$ in all these problems and the sum of the individual demands is always at least $k * a=\sum_{i \in K} y_{i}$. Similarly, if we define the counterpart problems $\tilde{P}_{0}, \tilde{P}_{1}, \tilde{P}_{2}, \ldots, \tilde{P}_{k}$ where $\tilde{P}_{i}=$ $\left(c ; \tilde{a}, \tilde{a}, \ldots, \tilde{a}, \tilde{y}_{i+1}, \tilde{y}_{i+2}, \ldots, \tilde{y}_{k-1}, \tilde{y}_{k}\right)$, then again all of them will be feasible.

Now, due to the separability lemma, we must have $\xi_{1}\left(P_{0}\right)=\xi_{1}\left(P_{1}\right)$. But then strong monotonicity and budget balancedness imply $\xi_{-1}\left(P_{0}\right)=\xi_{-1}\left(P_{1}\right)$. Thus, we have $\xi\left(P_{0}\right)=\xi\left(P_{1}\right)$. Using the same argument, we have $\xi\left(P_{i}\right)=\xi\left(P_{i+1}\right)$ and $\xi\left(\tilde{P}_{i}\right)=\xi\left(\tilde{P}_{i+1}\right)$ for all $0 \leq i \leq k-1$. Thus, we have $\xi\left(P_{0}\right)=\xi\left(P_{k}\right)$ and $\xi\left(\tilde{P}_{0}\right)=\xi\left(\tilde{P}_{k}\right)$ as desired.

Step 2: $\xi(c ; a, a, \ldots, a)=\xi(c ; \tilde{a}, \tilde{a}, \ldots ., \tilde{a})$

Proof:

Notice first that the feasibility of $(c ; a, a, \ldots, a) \& \xi(c ; \tilde{a}, \tilde{a}, \ldots ., \tilde{a})$ implies that any problem $(c ; \hat{a})$ where some of the $\hat{a}_{i}=a$ and other $\hat{a}_{i}=\tilde{a}$ is also feasible. Now, lemma 2 implies $\xi_{1}(c ; a, \tilde{a}, \ldots, \tilde{a})=\xi_{1}(c ; \tilde{a}, \tilde{a}, \ldots ., \tilde{a})$. Now, there can be three cases $-a<\tilde{a}, a>\tilde{a}$ or $a=\tilde{a}$. In the first two cases strong monotonicity and budget-balancedness imply $\xi_{-1}(c ; a, \tilde{a}, \ldots, \tilde{a})=\xi_{-1}(c ; \tilde{a}, \tilde{a}, \ldots ., \tilde{a})$ and we get $\xi(c ; a, \tilde{a}, \ldots, \tilde{a})=\xi(c ; \tilde{a}, \tilde{a}, \ldots ., \tilde{a})$. The third case trivially implies $\xi(c ; a, \tilde{a}, \ldots, \tilde{a})=$ $\xi(c ; \tilde{a}, \tilde{a}, \ldots ., \tilde{a})$ since it is the same problem so the solution must be the same. Similarly, we get $\xi(c ; \tilde{a}, \tilde{a}, \ldots ., \tilde{a})=\xi(c ; a, \tilde{a}, \ldots, \tilde{a})=\xi(c ; a, a, \tilde{a}, \ldots, \tilde{a})=\ldots \ldots .=$ $\xi(c ; a, a, \ldots, a)$.

6.3.2 2. $\Longrightarrow 1$.

\section{Proof.}


We know that $\xi$ PNI efficient graph implies that $\xi$ is efficient. We will prove that if $\xi$ PNI the efficient graph then $\xi$ is strongly monotonic. Consider a $\xi$ that PNI the efficient graph and a feasible problem $(c ; y)$ and assume without loss of generality that $y_{1}<y_{2}<\ldots .<y_{k}{ }^{6}$. Now, consider a graph as shown in figure 6 below.

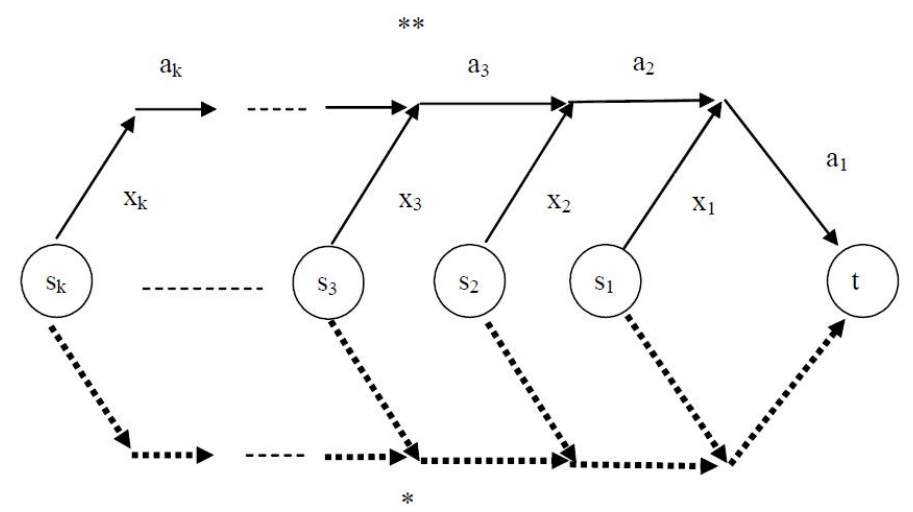

Figure 6: PNI implies SM.

Here every agent has two strategies- either use the path in the solid graph or use that in the dotted graph. Let's call the solid graph ** and the dotted graph *. Let $*$ be a small perturbation of $* *$ as following. The cost of path of an agent $j \neq i$ in both graphs is $y_{j}$. The cost of paths of agent $i$ in "**" and "*" are $y_{i}$ and $\tilde{y}_{i}$ where $\tilde{y}_{i}$ is in a neighborhood of $y_{i}$ and $\tilde{y}_{i}>y_{i}$ and $\left|\tilde{y}_{i}-y_{i}\right|<\min _{j, k \in K}\left|y_{j}-y_{k}\right|$. This restriction guarantees that the ranking will be preserved in the perturbed problem. Let the total cost of $* *$ and ${ }^{*}$ be $c-\epsilon$ and $c$ respectively. First, we will show that this graph generates all feasible problems $(c ; y)$. This happens if and only if the following system has a solution:

${ }^{6}$ The case of weak inequality will follow from the assumption of continuity on our method 


$$
\begin{aligned}
x_{1}+a_{1} & =y_{1} \\
x_{2}+a_{2}+a_{1} & =y_{2} \\
x_{3}+a_{3}+a_{2}+a_{1} & =y_{3} \\
& : \\
& : \\
x_{k}+a_{k}+a_{k-1}+\ldots+a_{1} & =y_{k} \\
\sum_{i=1}^{k} x_{i}+\sum_{i=1}^{k} a_{i} & =c \\
\forall i & \in K ; x_{i}, a_{i} \geq 0
\end{aligned}
$$

We use Farka's Lemma to prove that this system indeed has a solution:

From Farka's lemma we know that $A x=b ; x \geq 0$ has a solution if and only if $A^{T} z \geq 0 ; b^{T} z<0$ doesn't have a solution.

Here, the $(k+1) \times(2 k)$ matrix $A$, vector $x$ and vector $b$ are defined as follows:

$$
\begin{aligned}
A & =\left[\begin{array}{cccccccc}
1 & 0 & 0 & \ldots & 1 & 0 & 0 & \ldots . \\
0 & 1 & 0 & \ldots & 1 & 1 & 0 & \ldots . \\
\ldots & \ldots & \ldots & \ldots & \ldots & \ldots & \ldots . & \ldots . \\
1 & 1 & 1 & \ldots & 1 & 1 & 1 & \ldots .
\end{array}\right] \\
x & =\left[\begin{array}{llllllll}
x_{1} & x_{2} & \ldots & x_{k} & a_{1} & a_{2} & \ldots . & a_{k}
\end{array}\right]^{T} \\
b & =\left[\begin{array}{lllll}
y_{1} & y_{2} & \ldots & y_{k} & c
\end{array}\right]^{T}
\end{aligned}
$$

$A^{T} z \geq 0 ; b^{T} z<0$ gives the following $(2 k+1)$ inequalities;

$$
\begin{aligned}
& z_{1}+z_{2}+\ldots+z_{k+1} \geq 0 \\
& z_{2}+z_{3}+\ldots+z_{k+1} \geq 0
\end{aligned}
$$

$$
\begin{gathered}
z_{k}+z_{k+1} \geq 0 \\
z_{1}+z_{k+1} \geq 0 \\
z_{2}+z_{k+1} \geq 0 \\
: \\
z_{k}+z_{k+1} \geq 0
\end{gathered}
$$




$$
y_{1} z_{1}+y_{2} z_{2}+\ldots . .+y_{k} z_{k}+c z_{k+1}<0
$$

Now, do the following operation on the first $\mathrm{k}$ inequalities: $y_{1} \times(1)+\left(y_{2}-\right.$ $\left.y_{1}\right) \times(2)+\ldots \ldots+\left(y_{k}-y_{k-1}\right) \times(k)$, to get,

$$
y_{1} z_{1}+y_{2} z_{2}+\ldots . .+y_{k} z_{k}+y_{k} z_{k+1} \geq 0
$$

Now, for the inequalities $(2 \mathrm{k}+1)$ and $(2 \mathrm{k}+2)$ to be compatible, it must be the case that $z_{k+1}<0$. Let this be the case and let $(2 \mathrm{k}+2)$ and $(2 \mathrm{k}+1)$ hold. Then, $(2 \mathrm{k}+1)$ implies:

$$
y_{1} z_{1}+y_{2} z_{2}+\ldots .+y_{k} z_{k}+\left(\sum_{i \in K} y_{i}\right) z_{k+1}<0
$$

This is true because feasibility requires $\sum_{i \in K} y_{i} \geq c$. Now, if we do the following operation on inequalities $(k+1)$ through $(2 k): y_{1} \times(k+1)+y_{2} \times(k+$ $2)+\ldots .+y_{n} \times(2 k)$, then we get,

$$
y_{1} z_{1}+y_{2} z_{2}+\ldots .+y_{k} z_{k}+\left(\sum_{i \in K} y_{i}\right) z_{k+1} \geq 0
$$

which contradicts $(2 \mathrm{k}+3)$ to give us the desired result.

We now prove the strong monotonicity of $\xi$. Clearly, the efficiency of $\xi$ implies that ${ }^{* *}$ is a NE but since ${ }^{*}$ is a perturbation of $* *$, we will have ${ }^{*}$ as a NE for a perturbation small enough. The fact that $\xi$ PNI the efficient graph implies the following inequality

$$
\xi\left(c-\epsilon ; y_{i}, y_{-i}\right) \leq \xi\left(c ; \tilde{y}_{i}, y_{-i}\right)
$$

Using continuity we get,

$$
\xi\left(c ; y_{i}, y_{-i}\right) \leq \xi\left(c ; \tilde{y}_{i}, y_{-i}\right)
$$

Now consider a perturbation where every thing is exactly the same except ** costs $c+\epsilon$. Using the same argument of Pareto Nash implementability and continuity we get that

$$
\xi\left(c ; y_{i}, y_{-i}\right) \geq \xi\left(c ; \tilde{y}_{i}, y_{-i}\right)
$$

Thus we conclude that $\xi\left(c ; y_{i}, y_{-i}\right)=\xi\left(c ; \tilde{y}_{i}, y_{-i}\right)$ for $\tilde{y}_{i}$ in an open neighborhood of $y_{i}$. But repeatedly using the open neighborhood argument, show that this is true for any arbitrary $y_{i}$ and $\tilde{y}_{i}$ as long as $\left(c ; y_{i}, y_{-i}\right)$ and $\left(c ; \tilde{y}_{i}, y_{-i}\right)$ are both feasible. 


\subsection{3 $3 . \Longrightarrow 4$.}

Consider a continuous $\xi$ that implements the efficient graph in strong NE. Consider two arbitrary feasible problems $(c ; y)$ and $(c ; \tilde{y})$. We will prove that $\xi(c ; y)=\xi(c ; \tilde{y})=f(c)$. The monotonicity of $f$ comes from lemma 1 . Let $a=\frac{1}{k} \sum_{i \in K} y_{i}$ and $\tilde{a}=\frac{1}{k} \sum_{i \in K} \tilde{y}_{i}$. Assume without loss of generality that $y_{1} \leq y_{2} \leq y_{3} \leq \ldots \ldots \leq y_{k}$ and $\tilde{y}_{1} \leq \tilde{y}_{2} \leq \tilde{y}_{3} \leq \ldots \ldots \leq \tilde{y}_{k}$.

Step 1: $\xi(c ; y)=\xi(c ; a, a, \ldots, a)$ and $\xi(c ; \tilde{y})=\xi(c ; \tilde{a}, \tilde{a}, \ldots ., \tilde{a})$

Proof:

Consider the following problems: $P_{0}=(c ; y), P_{1}=\left(c ; a, y_{2}, y_{3}, \ldots y_{k}\right), P_{2}=$ $\left(c ; a, a, y_{3}, y_{4}, \ldots, y_{k}\right), \ldots \ldots \ldots, P_{k}=(c ; a, a, \ldots \ldots, a)$. Notice first that the feasibility of $P_{0}$ implies the feasibility of $P_{1}, P_{2}, \ldots, P_{k}$. This is true because the maximum of the demand profile doesn't go above $y_{k}$ in all these problems and the sum of the individual demands is always at least $k * a=\sum_{i \in K} y_{i}$. Similarly, if we define the counterpart problems $\tilde{P}_{0}, \tilde{P}_{1}, \tilde{P}_{2}, \ldots, \tilde{P}_{k}$ where $\tilde{P}_{i}=$ $\left(c ; \tilde{a}, \tilde{a}, \ldots, \tilde{a}, \tilde{y}_{i+1}, \tilde{y}_{i+2}, \ldots, \tilde{y}_{k-1}, \tilde{y}_{k}\right)$, then again all of them will be feasible.

Now, due to the separability lemma, we must have $\xi_{1}\left(P_{0}\right)=\xi_{1}\left(P_{1}\right)$. Also, strong Nash implementability implies that $\xi_{-1}\left(P_{0}\right)=\xi_{-1}\left(P_{1}\right)$. To see this, suppose that it is not the case and for some agent $j \neq 1$, we have $\xi_{j}\left(P_{0}\right) \neq$ $\xi_{j}\left(P_{1}\right)$. Assume without loss of generality that $\xi_{j}\left(P_{0}\right)<\xi_{j}\left(P_{1}\right)$. This means $\exists \grave{j} \in K \backslash\{1, j\}$ s.t., $\xi_{j}\left(P_{0}\right)>\xi_{j}\left(P_{1}\right)$, because of budget balancedness. Consider a network where all the agents $2,3, \ldots, k$ have just one strategy which costs $y_{2}, y_{3}, \ldots, y_{k}$ and agent 1 has two strategies, where one of them costs $y_{1}$ and the other costs $a$. In both the cases, the total cost of the network is $c$. Thus one of the configurations generates the problem $P_{0}$ and the other $P_{1}$. Now both the configurations of the network are efficient and therefore at least one of them must be a strong NE under $\xi$. But clearly none of them is a strong NE. From $P_{1}$ the group $\{1, j\}$ has a profitable deviation and from $P_{0}$ the group $\{1, \grave{j}\}$. Thus, we have $\xi\left(P_{0}\right)=\xi\left(P_{1}\right)$. Using the same argument we have $\xi\left(P_{i}\right)=\xi\left(P_{i+1}\right)$ and $\xi\left(\tilde{P}_{i}\right)=\xi\left(\tilde{P}_{i+1}\right)$ for all $0 \leq i \leq k-1$. Thus, we have $\xi\left(P_{0}\right)=\xi\left(P_{k}\right)$ and $\xi\left(\tilde{P}_{0}\right)=\xi\left(\tilde{P}_{k}\right)$ as desired.

Step 2: $\xi(c ; a, a, \ldots, a)=\xi(c ; \tilde{a}, \tilde{a}, \ldots ., \tilde{a})$

Proof:

Notice first that the feasibility of $(c ; a, a, \ldots, a) \& \xi(c ; \tilde{a}, \tilde{a}, \ldots ., \tilde{a})$ implies that any problem $(c ; \hat{a})$ where some of the $\hat{a}_{i}=a$ and other $\hat{a}_{i}=\tilde{a}$ is also feasible. Now, lemma 2 implies $\xi_{1}(c ; a, \tilde{a}, \ldots, \tilde{a})=\xi_{1}(c ; \tilde{a}, \tilde{a}, \ldots ., \tilde{a})$. And again, the strong Nash implementability implies $\xi_{-1}(c ; a, \tilde{a}, \ldots, \tilde{a})=\xi_{-1}(c ; \tilde{a}, \tilde{a}, \ldots ., \tilde{a})$. The proof of this statement is analogous to the one in step 1 . Thus we have $\xi(c ; a, \tilde{a}, \ldots, \tilde{a})=$ $\xi(c ; \tilde{a}, \tilde{a}, \ldots ., \tilde{a})$. Similarly, we get

$$
\xi(c ; \tilde{a}, \tilde{a}, \ldots ., \tilde{a})=\xi(c ; a, \tilde{a}, \ldots, \tilde{a})=\xi(c ; a, a, \tilde{a}, \ldots, \tilde{a})=\ldots \ldots . .=\xi(c ; a, a, \ldots, a) .
$$

The results "4. $\Longrightarrow 1 ., "$ "4. $\Longrightarrow 2$ " and "4. $\Longrightarrow 3$ " are straightforward 
and the proof is omitted.

\subsection{Proof of Theorem 2}

Proof. The "if" part is clear. For, "only if" consider an arbitrary feasible problem $(c ; y)$. Assume without loss of generality that $y_{1} \geq y_{2} \geq y_{3} \geq \ldots \geq y_{k}$. Let $a=\frac{1}{k} \sum_{i=1}^{k} y_{i}$. Consider a problem $(c ; a, a, \ldots ., a)$ and suppose that $\xi$ is continuous, efficient and satisfies ETE. Notice that the feasibility of $(c ; y)$ implies the feasibility of $(c ; a, a, \ldots, a)$ and any other problem $(c ; \hat{y})$ where $\hat{y}_{i}=y_{i}$ for all $i \in\{1,2, \ldots, l\}$ and $\hat{y}_{i}=a$ for all $i \in\{l, l+1, \ldots, k-1, k\}$. Now, the ETE property of $\xi$ implies

$$
\xi(c ; a, a, \ldots, a)=(c / k, c / k, \ldots ., c / k)
$$

Using lemma 2 and applying ETE again we get,

$$
\xi\left(c ; y_{1}, a, \ldots, a\right)=(c / k, c / k, \ldots ., c / k)
$$

Now again applying lemma 2 and ETE we have,

$$
\xi\left(c ; y_{1}, y_{2}, a, a, \ldots ., a\right)=\left(x_{1}, c / k, x, x, \ldots ., x\right)
$$

But if we change the ordering of $1 \& 2$ while arriving at the above profile then we should have,

$$
\xi\left(c ; y_{1}, y_{2}, a, a, \ldots, a\right)=\left(c / k, x_{2}, x, x, \ldots, x\right)
$$

But, since the ordering is immaterial, we must have that $x_{1}, x_{2}, x=c / k$. And thus we have,

$$
\xi\left(c ; y_{1}, y_{2}, a, a, \ldots ., a\right)=(c / k, c / k, \ldots ., c / k)
$$

Repeating the same argument, we conclude that $\xi(c ; y)=(c / k, c / k, \ldots, c / k)$

\section{Proof of Lemma 2}

We show the existence of equilibrium for PR.

Proof. We prove a stronger property, which is that the best response (br) dynamics (one agent at a time) of any arbitrary fixed ordering of agents converges to a NE, no matter where we start the br dynamics from. Suppose, on the contrary, that for some fixed ordering of agents the br dynamics from some point " $s$ " does not converge. This means that there is a cycle of a finite length $l-$ $s(1) \rightarrow s(2) \rightarrow s(3) \rightarrow \ldots \ldots . . \rightarrow s(l) \rightarrow s(1)$. Say, without loss of generality, that this cycle includes deviations by the set of agents $M=\{1,2, \ldots, m\} \subseteq K$. The strategy of agents in $K / M$ is fixed at $s^{-M}$. Notice that $l$ is at least as big as $2 m$. This is so because after the $l$ best responses, we arrive at the original strategy profile i.e., $s(1)$. Every agent in $M$ is a part of the cycle, which in turn means 
that they change their strategy at least once. Therefore, it must be the case that every agent in $M$ takes its turn at least twice so that they reach the original profile, i.e., $s(1)$. Let's assume that agent $i \in M$ takes its turn in the br dynamics $n_{i}>1$ number of times so that $\sum_{i \in M} n_{i}=l$. Let the strategies played by the agent $i$ in the cycle be $s^{i ; 1}, s^{i ; 2}, \ldots, s^{i ; n_{i}}, s^{i ; 1}$ and so on. Let's call the agent who takes his turn of br in the movement from $s_{t}$ to $s_{t+1}$ agent $a_{t}$. Therefore, $s(1)=\left(s^{1 ; 1}, s^{2 ; 1}, \ldots, s^{m ; 1}, s^{-M}\right), s(2)=\left(s^{a_{1} ; 2}, s_{-a_{1}}(1)\right), s(3)=\left(s^{a_{2} ; 2}, s_{-a_{2}}(2)\right)$,

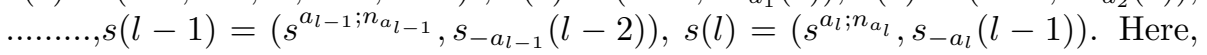
we use the standard notation where $s_{-i}(t)$ represents the strategy profile of $K \backslash\{i\}$ fixed at that in $s(t)$. We abuse the notation and say that the cost of $s^{p ; i}$ is equal to $s^{p ; i}$. Here the cost of the network formed by the strategy profile $s(i)=C\left(G_{s(i)}\right)$. Now, $\xi_{j}^{p r}\left(C\left(G_{s(i)}\right) ; s(i)\right)=s^{j ; p} A_{i}$ where $A_{i}$ is fixed for any particular $s(i)$ and $s^{j ; p}$ represents the strategy of agent $j$ in $s(i)$. The fixed $A_{i}$ for an $s(i)$ is the ratio of $C\left(G_{s(i)}\right)$ to the sum of the costs of individual paths in $s(i)$.

Now every step of the cycle corresponds to an inequality which we will present as follows:

Step 1: $s(1) \rightarrow s(2) \Longrightarrow$

$$
s^{a_{1} ; 2} \times A_{2}<s^{a_{1} ; 1} \times A_{1}
$$

Step 2: $s(2) \rightarrow s(3) \Longrightarrow$

$$
s^{a_{2} ; 2} \times A_{3}<s^{a_{2} ; 1} \times A_{2}
$$

Step 3: $s(3) \rightarrow s(4) \Longrightarrow$

$$
s^{a_{3} ; t} \times A_{4}<s^{a_{3} ; t-1} \times A_{3} ; t=\left\{\begin{array}{c}
3 \text { if } a_{3}=a_{1} \\
2 \text { otherwise }
\end{array}\right\}
$$

Step p: $s(p) \rightarrow s(p+1) \Longrightarrow$

$$
s^{a_{p} ; t} \times A_{p+1}<s^{a_{p} ; t-1} \times A_{p} ; t \in\left\{1,2, \ldots, n_{a_{p}}\right\}
$$

Step l: $s_{l} \rightarrow s_{1} \Longrightarrow$

$$
s^{a_{l} ; n_{a_{l}}} \times A_{1}<s^{a_{l} ; n_{a_{l}}-1} \times A_{l}
$$

If we multiply the systems $(2),(3), \ldots$, (l) together ${ }^{7}$, then everything else cancels out and we are left with $s^{a_{1} ; 2} \times A_{2}>s^{a_{1} ; 1} \times A_{1}$, which contradicts the

\footnotetext{
${ }^{7}$ Notice, we can do that since everything here is positive
} 
inequality (1). Therefore, we conclude that there cannot be any cycle regardless what ordering of agents and what initial point we follow for the best response dynamics.

\section{Proof of Theorem 3}

\subsection{Any AEM meets WPNI}

We start from an AEM $\varphi$ determined by the functions $f^{1}, \ldots, f^{k}$.

Let $\left.g^{i}=\left(f^{i}\right)^{(}-1\right)$ the inverse of $f^{i}$. Without loss of generality, we assume $g^{1}\left(s^{1}\right) \leq \cdots \leq g^{k}\left(s^{k}\right)$, where $s_{i}$ is the cost of the stand-alone $S^{i}$ of agent $i$.

Step 1. For any Nash equilibrium there is an index $m$ and $\lambda^{*}$ such that

i. $\varphi_{i}=s^{i}$ for $i=1, \ldots, m$

ii. $\varphi_{h}=f^{i}\left(\lambda^{*}\right)$ for $h>m$.

iii. $g^{m}\left(s^{m}\right)<\lambda^{*} \leq g^{m+1}\left(s^{m+1}\right)$

Proof. Consider an equilibrium $X=\left(X^{1}, \ldots, X^{k}\right)$ and let $\lambda^{*}$ such that $\sum_{i} \min \left\{C\left(X^{i}\right), f^{i}\left(\lambda^{*}\right)\right\}=C\left(X^{1}, \ldots, X^{k}\right)$.

If agent $j$ is such that $\varphi_{j}<f^{j}\left(\lambda^{*}\right)$, then $\varphi_{j}=s^{j}$. To see this, since $\varphi_{j}=$ $\min \left\{C\left(X^{j}\right), f^{i}\left(\lambda^{*}\right)\right\}<\lambda^{*}$, then $\varphi_{j}=C\left(X^{j}\right)$. Since $\left.C\left(X^{j}\right)\right) \geq s^{j}$, then agent $j$ can deviate to his stand-alone $S^{j}$ whenever $\left.C\left(X^{j}\right)\right)>s^{j}$, and guarantee a cost-share not larger than $s^{j}$. Therefore at equilibrium $\varphi_{j}=s^{j}$.

Since $g^{1}\left(s^{1}\right) \leq \cdots \leq g^{k}\left(s^{k}\right)$, then there is $m$ such that $i$ and $i i$ is satisfied. We now show that iii must be satisfied.

Assume that $\lambda^{*}>g^{m+1}\left(s^{m+1}\right)$ then $f^{m+1}\left(\lambda^{*}\right)>s^{m+1}$, thus $\varphi_{m+1}>$ $f^{m+1}\left(s^{m+1}\right)$. Thus agent $m+1$ can profit by deviating from $X$ by selecting $S^{m+1}$.

Finally, since $C\left(X^{i}\right)=s^{i}$ for $i=1, \ldots, m$, and $\varphi_{i}=s^{i}$, then $s^{i}<f^{i}\left(\lambda^{*}\right)$ thus $g^{i}\left(s^{i}\right)<\lambda^{*}$.

Step 2. $\varphi$ Pareto ranks the equilibriums.

Proof.

Consider any two equilibriums $X=\left(X^{1}, \ldots, X^{k}\right)$ and $\tilde{X}=\left(\tilde{X}^{1}, \ldots, \tilde{X}^{k}\right)$. Let $(m, \lambda)$ and $(\tilde{m}, \tilde{\lambda})$ the values given by step 1 for equilibrium $X$ and $\tilde{X}$ respectively.

If $m<\tilde{m}$, then $\lambda<\tilde{\lambda}$, since $g^{m}\left(s^{m}\right)<\lambda \leq g^{m+1}\left(s^{m+1}\right) \leq g^{\tilde{m}+1}\left(s^{\tilde{m}+1}\right)<\tilde{\lambda}$.

Hence, by step 1 , agents $\{1, \ldots, m\}$ are indifferent between both equilibriums and agents $\{m+1, \ldots k\}$ prefer equilibrium $X$ to $\tilde{X}$.

On the other hand, if $m=\tilde{m}$, then agents $\{1, \ldots, m\}$ are indifferent between both equilibriums, and agents $\{m+1, \ldots k\}$ rank the equilibrium depending on whether $\lambda<\tilde{\lambda}$ or vice versa. 


\subsection{WPNI implies AEG}

We start the proof for two agents.

\subsubsection{Proof for two agents}

Step 1. The mechanisms satisfy truncation, that is $\varphi\left[c ; y_{1}, y_{2}\right]=\varphi\left[c ; \tilde{y}_{1}, \tilde{y}_{2}\right]$ for any $\left(\tilde{y}_{1}, \tilde{y}_{2}\right) \geq \varphi\left[c ; y_{1}, y_{2}\right]$.

Proof.

Consider a feasible profile $\left(c ; y_{1}, y_{2}\right)$ such that $y_{1}+y_{2}>c$. Let $\left(p_{1}, p_{2}\right)$ such that $\varphi\left(c ; y_{1}, y_{2}\right)=\left(p_{1}, p_{2}\right)$ and assume without loss of generality that $p_{1}<y_{1}$. Consider $p_{1}<\tilde{y}_{1}<y_{1}$.

Construct networks depicted in figure 7 such that agent 1 has two strategies with costs $y_{1}$ and $\tilde{y}_{1}$, agent 2 also has two strategies with the same cost $y_{2}$, and the cost of the networks are $\left(c+\epsilon ; y_{1}, y_{2}\right)$ and $\left(c ; \tilde{y}_{1}, y_{2}\right)$.

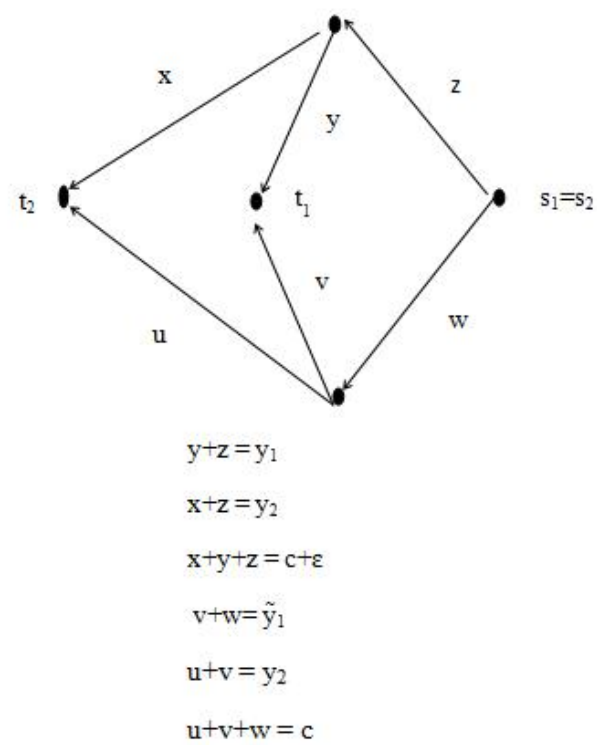

Figure 7: WPNI implies AEG (part 1).

Clearly the graphs that generate $\left(c+\epsilon ; y_{1}, y_{2}\right)$ and $\left(c ; \tilde{y}_{1}, y_{2}\right)$ are a Nash equilibrium for small $\epsilon$.

By WPNI: $\varphi\left(c+\epsilon ; y_{1}, y_{2}\right) \geq\left(c ; \tilde{y}_{1}, y_{2}\right)$.

As $\epsilon$ tends to zero, and using continuity:

$$
\varphi\left(c ; y_{1}, y_{2}\right) \geq \varphi\left(c ; \tilde{y}_{1}, y_{2}\right)
$$


Similarly, consider the network in figure 8 such that agents 1 has two strategies with costs $y_{1}$ and $\tilde{y}_{1}$, agent 2 also has two strategies with the same cost $y_{2}$, and the cost of the networks are $\left(c ; y_{1}, y_{2}\right)$ and $\left(c+\epsilon ; \tilde{y}_{1}, y_{2}\right)$.

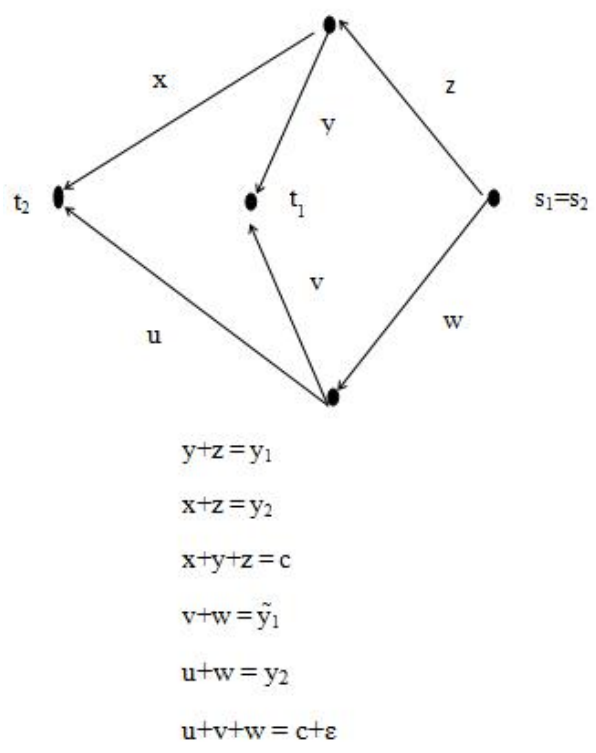

Figure 8: WPNI implies AEG (part 2).

Clearly those two graphs are Nash equilibriums for small $\epsilon$.

By WPNI: $\varphi\left(c+\epsilon ; \tilde{y}_{1}, y_{2}\right) \geq \varphi\left(c ; y_{1}, y_{2}\right)$.

As $\epsilon$ tends to zero, and using continuity:

$$
\varphi\left(c ; \tilde{y}_{1}, y_{2}\right) \geq \varphi\left(c ; y_{1}, y_{2}\right) .
$$

by equations 9 and 10 :

$$
\varphi\left(c ; \tilde{y}_{1}, y_{2}\right)=\varphi\left(c ; y_{1}, y_{2}\right) .
$$

For any $c>0$, let $g(c)=\varphi[c ; c, c]$.

Step 2. $\varphi\left[c ; y_{1}, y_{2}\right]=g(c)$ if $\left(y_{1}, y_{2}\right) \geq g(c) ;=\left(y_{1}, c-y_{1}\right)$ if $y_{1}<g_{1}(c)$; $=\left(c-y_{2}, y_{2}\right)$ if $y_{2}<g_{2}(c)$.

Proof.

By step 1 and continuity, $\varphi\left[c ; y_{1}, y_{2}\right]=g(c)$ if $\left(y_{1}, y_{2}\right) \geq g(c)$.

Consider $y=\left(y_{1}, c\right)$ such that $y_{1}<g_{1}(c)$; and let $\left(p_{1}, p_{2}\right)=\varphi\left[c ; y_{1}, c\right]$. Assume that $p_{1}<y_{1}$.

By continuity, $\varphi_{1}\left(y_{1}+\epsilon, c\right) \rightarrow p_{1}$ as $\epsilon$ tends to zero. Let $\tilde{\epsilon}$ by such that $\tilde{p}_{1}=\varphi_{1}\left(y_{1}+\tilde{\epsilon}, c\right)<y_{1}$. 
Consider the demand $\left(\frac{\tilde{p}_{1}+y_{1}}{2}, c\right)$, by truncation $\varphi\left(c ; \frac{\tilde{p}_{1}+y_{1}}{2}, \tilde{y}_{2}\right)=\left(\tilde{p}_{1}, c-\tilde{p}_{1}\right)$, for any $y_{2}>c-p_{1}$.

Similarly, $\varphi\left(c ; \frac{\tilde{p}_{1}+y_{1}}{2}, \tilde{y}_{2}\right)=\left(p_{1}, c-p_{1}\right)$, which is a contradiction.

By truncation, $\varphi_{1}\left(y_{1}+\tilde{\epsilon}, c\right)<y_{1}$.

Step 3. The mechanism should be weakly monotonic at the truncation point. That is $g(c)<g(\tilde{c})$ for $c<\tilde{c}$.

Proof.

Suppose that the mechanism is not weakly monotonic at the truncation point. Then, for any small $\epsilon$ we can find $c$ and $c+\epsilon$ such that $g_{2}(c)>g_{2}(c+\epsilon)$ and $g_{1}(c)>g_{1}(c+\epsilon)$ (or vice versa).

Pick small $\epsilon$ and $b>>\max \{g(c), g(c+\epsilon)\}$ and $c>b_{1}+b_{2}$.

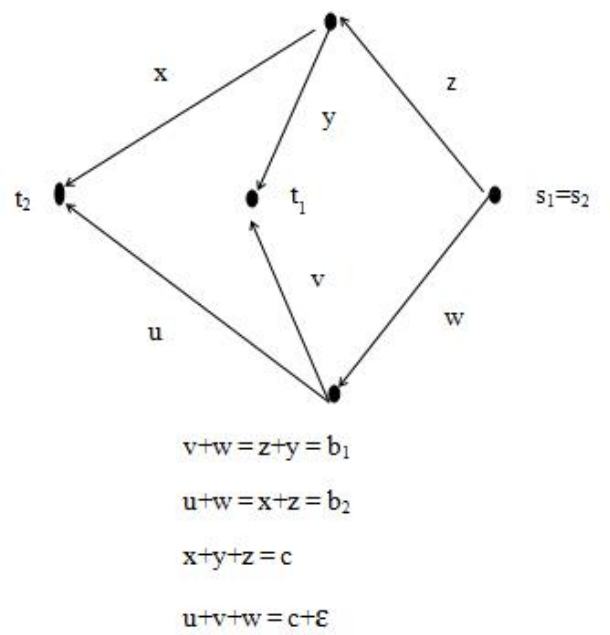

Figure 9: Monotonicy at truncation point.

Consider the network depicted in figure 9 such that every agent has two strategies, of equal cost $b_{1}$ and $b_{2}$, and generate problems $\left(c ; b_{1}, b_{2}\right)$ and $(c+$ $\left.\epsilon ; b_{1}, b_{2}\right)$. Clearly there are only two equilibria with costs $c$ and $c+\epsilon$ but they are not Pareto ranked since $\varphi\left(c ; b_{1}, b_{2}\right)=g(c)$ and $\varphi\left(c+\epsilon ; b_{1}, b_{2}\right)=g(c+\epsilon)$.

Step 4 . The mechanism can be represented by the above functions.

Proof. Consider the AEM represented by the functions $g_{i}(c)$ as above. It is easy to show that this mechanism generates the mechanism as above.

Indeed, consider $(c ; y)$ feasible. Then If $\left(y_{1}, y_{2}\right) \geq g(c)$, then $\varphi(c ; y)=g(c)$. If $y_{1}<g_{1}(c)$, then by truncation $\varphi_{1}(c ; y)=g_{1}(c)$ and $\varphi_{2}(c ; y)=c-g_{1}(c)$.

If $y_{2}<g_{2}(c)$, then by truncation $\varphi_{2}(c ; y)=g_{2}(c)$ and $\varphi_{1}(c ; y)=c-g_{2}(c)$. 


\subsubsection{Extension to more than two agents}

Proof. Consider any Parametric solution with $k$ agents, $k>2$. We can replicate the above arguments for a network of any two agents $\{i, j\}$ by setting $y_{l}=0$ for $l \neq i, j$ (demanding independent demands with cost zero). Thus, by the previous case, $F_{i}\left(\lambda, y_{i}\right)=\min \left\{y_{i}, g_{i}(\lambda)\right\}$ for some non-decreasing function $g_{i}(\lambda)$.

\section{$9 \quad$ Proof of Corollary 4}

\section{1 $\operatorname{POS}(E G)=H(k)$}

Consider the efficient profile $P=\left(P^{1}, \ldots, P^{k}\right)$ with cost $c^{*}$. Assume without loss of generality that $C\left(P^{1}\right) \geq C\left(P^{2}\right) \geq \cdots \geq C\left(P^{k}\right)$. Let $S^{i}$ the stand-alone path of agent $i$ with cost $s^{i}$.

Let $p=E G\left(c^{*} ; C\left(P^{1}\right), C\left(P^{2}\right), \ldots, C\left(P^{k}\right)\right)$. Clearly $p_{1} \geq p_{2} \geq \cdots \geq p_{k}$, and $p_{i} \leq \frac{c^{*}}{i}$ for $i=1, \ldots, k$.

Let $\lambda^{*}$ and $m$ be such that $p_{k} \leq p_{k-1} \leq \cdots \leq p_{m+1}<\lambda^{*}=p_{m} \cdots=p_{1}$.

Let $\tilde{K}=\left\{i \mid s^{i}<\lambda^{*}\right\}$. That is, $\tilde{K}$ is the set of agents with stand-alone less than $\lambda^{*}$. Consider the profile $Q=\left(P_{(\tilde{K})^{c}}, S_{\tilde{K}}\right)$, that is the strategy from each agents in $\tilde{K}$ is replaced by his stand-alone path.

Clearly, if $i>m$ then $i \in \tilde{K}$, since $s^{i} \leq p_{i}<\lambda^{*}$. Therefore $Q$ contains at least all the agents who are paying their demand at $P$, but might include others.

Let $\tilde{k}=|\tilde{K}|$ the cardinality of $\tilde{K}$. First, notice that

$$
C\left(S_{\tilde{K}}\right) \leq(\tilde{k}-m) \lambda^{*}+s^{m+1}+\cdots+s^{k} \leq(\tilde{k}-m) \frac{c^{*}}{m}+\frac{c^{*}}{m+1}+\cdots+\frac{c^{*}}{k} .
$$

Therefore $C\left(S_{(\tilde{K})}\right) \leq \frac{c^{*}}{k-\tilde{k}+1}+\cdots+\frac{c^{*}}{k}$.

Hence $C(Q) \leq c^{*}+C\left(S_{(\tilde{K})}\right) \leq c^{*}+\frac{c^{*}}{k-\tilde{k}+1}+\cdots+\frac{c^{*}}{k}$.

We repeat the above algorithm consecutively to the profile $Q$. That is, we find $\lambda$ and move all the agent with stand-alone cost less than $\lambda$ to their standalone path. Since there is at most $k$ agents, this algorithm finishes in at most $k$ steps. Let $R$ the final profile of this algorithm.

From the above arguments, $C(R) \leq H(k) c^{*}$.

Let $\tilde{\lambda}$ the solution to the problem $E G\left(C(R) ; C\left(R_{1}\right), \ldots, C\left(R_{k}\right)\right)$.

If $E G_{i}\left(C(R) ; C\left(R_{1}\right), \ldots, C\left(R_{k}\right)\right)=\tilde{\lambda}$ then $s_{i} \geq \tilde{\lambda}$.

On the other hand, if $E G_{i}\left(C(R) ; C\left(R_{1}\right), \ldots, C\left(R_{k}\right)\right)<\tilde{\lambda}$ then $R^{i}=S^{i}$.

Similarly to the existence of equilibrium for AEM, the best reply tattonment would converge to an equilibrium starting from the profile $R$, since $\lambda$ and the cost would decrease at every step.

Indeed, if an agent is paying his stand alone, the only way to decrease his payment is by increasing his demand, and thus decreasing his cost. Therefore $\tilde{\lambda}$ should decrease. At his best reply, his stand-alone should be larger than the new $\lambda$. 
On the contrary, if an agent is paying $\tilde{\lambda}$, then his best reply should decrease $\tilde{\lambda}$ because his stand-alone is larger than $\tilde{\lambda}$.

\subsection{For any AEM $\xi, \xi \neq E G, \operatorname{PoS}(\xi)>H(k)$}

Proof. Consider the AEM mechanism $\xi$ generated by the functions $f^{1}, \ldots, f^{k}$. Since $\xi \neq E G$, then there is $i, j$ such that $f^{i} \neq f^{j}$.

Let $\lambda^{*}$ be such that $f^{i}\left(\lambda^{*}\right) \neq f^{j}\left(\lambda^{*}\right)$, and $c^{*}$ such that $c^{*}=f^{1}\left(\lambda^{*}\right)+\cdots+$ $f^{k}\left(\lambda^{*}\right)$.

There is an agent $l$ such that $f^{l}\left(\lambda^{*}\right)>\frac{c^{*}}{k}$, without loss of generality, assume such agent is agent $k$. That is, $f^{k}\left(\lambda^{*}\right)>\frac{c^{*}}{k}$. Let $\varphi_{k}^{*}=\varphi_{k}\left[c^{*} ; c^{*}, \ldots, c^{*}\right]=$ $f^{k}\left(\lambda^{*}\right)>\frac{c^{*}}{k}$.

Consider the problem $\left[c^{*}+f^{k}\left(\lambda^{*}\right) ; c^{*}, c^{*}, \ldots, f^{k}\left(\lambda^{*}\right)\right]$. Since

$$
\varphi_{k}\left[c^{*}+f^{k}\left(\lambda^{*}\right) ; c^{*}, c^{*}, \ldots, f^{k}\left(\lambda^{*}\right)\right] \leq f^{k}\left(\lambda^{*}\right),
$$

then there is an agent $l, l \neq k$, such that

$$
\varphi_{l}\left[c^{*}+f^{k}\left(\lambda^{*}\right) ; c^{*}, c^{*}, \ldots, f^{k}\left(\lambda^{*}\right)\right] \geq \frac{c^{*}}{k-1} .
$$

Without loss of generality, assume such agent is agent $k-1$. Let

$$
\varphi_{k-1}^{*}=\varphi_{k-1}\left[c^{*}+f^{k}\left(\lambda^{*}\right) ; c^{*}, c^{*}, \ldots, f^{k}\left(\lambda^{*}\right)\right],
$$

thus $\varphi_{k-1}^{*} \geq \frac{c^{*}}{k-1}$.

Consider the problem

$$
\left[c^{*}+\varphi_{k-1}^{*}+f^{k}\left(\lambda^{*}\right) ; c^{*}, c^{*}, \ldots, c^{*}, \varphi_{k-1}^{*}, f^{k}\left(\lambda^{*}\right)\right] .
$$

Since

$$
\varphi_{k}\left[c^{*}+\varphi_{k-1}^{*}+f^{k}\left(\lambda^{*}\right) ; c^{*}, c^{*}, \ldots, c^{*}, \varphi_{k-1}^{*}, f^{k}\left(\lambda^{*}\right)\right] \leq f^{k}\left(\lambda^{*}\right)
$$

and

$$
\varphi_{k-1}\left[c^{*}+\varphi_{k-1}^{*}+f^{k}\left(\lambda^{*}\right) ; c^{*}, c^{*}, \ldots, c^{*}, \varphi_{k-1}^{*}, f^{k}\left(\lambda^{*}\right)\right] \leq \varphi_{k-1}^{*} .
$$

Then there is an agent $l$ such that

$$
\varphi_{l}\left[c^{*}+\varphi_{k-1}^{*}+f^{k}\left(\lambda^{*}\right) ; c^{*}, c^{*}, \ldots, c^{*}, \varphi_{k-1}^{*}, f^{k}\left(\lambda^{*}\right)\right] \geq \frac{c^{*}}{k-2} .
$$

Without loss of generality, assume such agent is agent $k-2$. Let

$$
\varphi_{k-2}^{*}=\varphi_{k-2}\left[c^{*}+\varphi_{k-1}^{*}+f^{k}\left(\lambda^{*}\right) ; c^{*}, c^{*}, \ldots, c^{*}, \varphi_{k-1}^{*}, f^{k}\left(\lambda^{*}\right)\right],
$$

thus $\varphi_{k-2}^{*} \geq \frac{c^{*}}{k-2}$.

Continuing the same way, at step $i$, consider the problem

$$
\left[c^{*}+\varphi_{i+1}^{*}+\cdots+\varphi_{k-1}^{*}+f^{k}\left(\lambda^{*}\right) ; c^{*}, c^{*}, \ldots, c^{*}, \varphi_{i+1}^{*}, \ldots, \varphi_{k-1}^{*}, f^{k}\left(\lambda^{*}\right)\right] .
$$


Since

$\varphi_{k}\left[c^{*}+\varphi_{i+1}^{*}+\cdots+\varphi_{k-1}^{*}+f^{k}\left(\lambda^{*}\right) ; c^{*}, c^{*}, \ldots, c^{*}, \varphi_{i+1}^{*}, \ldots, \varphi_{k-1}^{*}, f^{k}\left(\lambda^{*}\right)\right] \leq f^{k}\left(\lambda^{*}\right)$

and

$\varphi_{j}\left[c^{*}+\varphi_{i+1}^{*}+\cdots+\varphi_{k-1}^{*}+f^{k}\left(\lambda^{*}\right) ; c^{*}, c^{*}, \ldots, c^{*}, \varphi_{i+1}^{*}, \ldots, \varphi_{k-1}^{*}, f^{k}\left(\lambda^{*}\right)\right] \leq \varphi_{j}^{*}$,

for $j=k-1, \ldots i+1$. Then there is an agent $l$ such that

$\varphi_{l}\left[c^{*}+\varphi_{i+1}^{*}+\cdots+\varphi_{k-1}^{*}+f^{k}\left(\lambda^{*}\right) ; c^{*}, c^{*}, \ldots, c^{*}, \varphi_{i+1}^{*}, \ldots, \varphi_{k-1}^{*}, f^{k}\left(\lambda^{*}\right)\right] \geq \frac{c^{*}}{i}$.

Without loss of generality, assume such agent is agent $i$. Let

$\varphi_{i}^{*}=\varphi_{i}\left[c^{*}+\varphi_{i+1}^{*}+\cdots+\varphi_{k-1}^{*}+f^{k}\left(\lambda^{*}\right) ; c^{*}, c^{*}, \ldots, c^{*}, \varphi_{i+1}^{*}, \ldots, \varphi_{k-1}^{*}, f^{k}\left(\lambda^{*}\right)\right]$.

Thus, $\varphi_{i}^{*} \geq \frac{c^{*}}{i}$.

Consider the network in figure 10 .

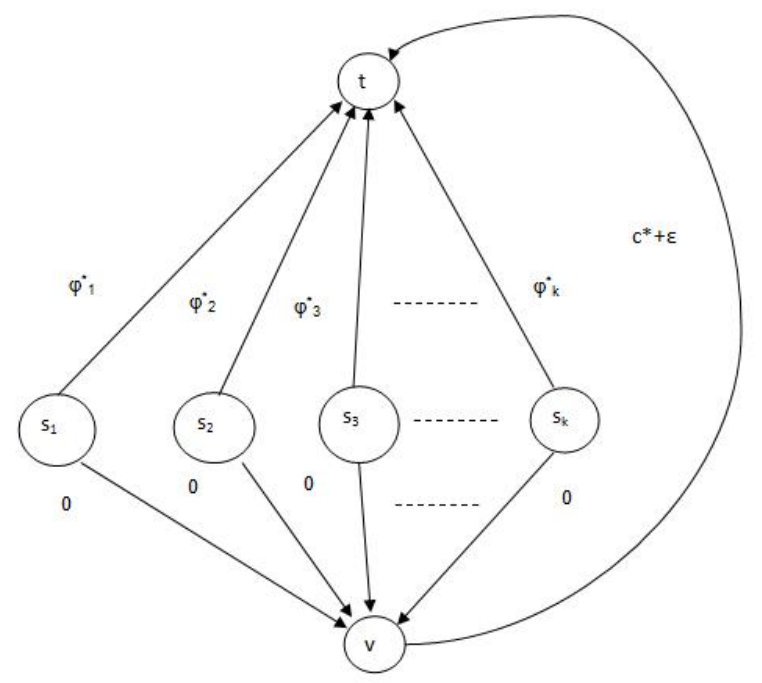

Figure 10: Optimality of the EG mechanism.

Since $\varphi_{i}^{*} \geq \frac{c^{*}}{i}$ for $i=1, \ldots, k-1$ and $\varphi_{k}^{*}>\frac{c^{*}}{k}$, then the only equilibrium is where agent $i$ demands the link $\left(s_{i}, t\right)$ with $\operatorname{cost} \varphi_{i}^{*}$. This equilibriumis inefficient and has a cost equal to $\sum_{i=1}^{k} \varphi_{i}^{*}>H(k)\left(c^{*}+\epsilon\right)$, for small $\epsilon$. 


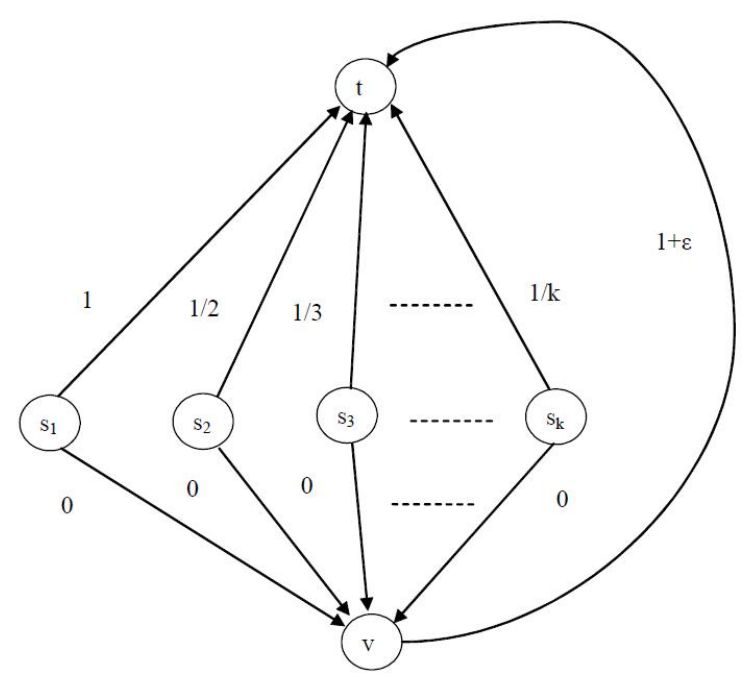

Figure 11: Incompatibility of EFF and IR

\subsection{Any IR mechanism has a PoS at least $H(k)$}

\section{Proof.}

We show by an example that any individually rational cost-sharing rule must have a PoS of at least $H(k)$. Consider a situation as shown in figure 11. Here, every agent $i$ has two strategies- either connect its demand nodes directly where the cost of the path is $1 / i$ or connect through the path where link costs are 0 and $1+\epsilon$. Consider any arbitrary cost-sharing method $\xi$ that satisfies individual rationality. We will show that if there exist an equilibrium, then this is where every agent is using its direct path to $t$. We prove this by contradiction.

Case 1. Assume all the agents use a free link to $v$ and then the common link of cost $1+\epsilon$ to $t$. But then at least one of the agents must be paying more than $1 / k$. Let's assume that this agent is the $k$-th agent in some configuration ${ }^{8}$ of the graph. Then he will have a profitable deviation to go to the direct link of cost $1 / k$ under any individually rational rule.

Case 2. Assume $s$ agents are using their direct link and $k-s$ agents are sharing the common link to $v$. Then it follows from the individual rationality of the $s$ agents that at least one of the remaining $k-s$ agents must be paying more than $1 /(k-s)$. Notice that in this case there exists an unused direct link, say, $s_{j} \rightarrow t$, of cost $1 / s_{j}$ which is at most $1 /(k-s)$. Now in some configuration of the graph, agent $j$ will be the agent who is paying the above mentioned amount of more than $1 /(k-s)$ and thus he would like to deviate.

We have just shown that no configuration different than the direct connection

\footnotetext{
${ }^{8}$ It is important to note that just one such configuration is enough, since PoS is a measure of the performance of the best NE in the worst case example.
} 
is a Nash equilibrium. If the equilibrium exists, then it must be the direct connection and has a cost equal to $H(k)$, whereas the efficient graph has a cost equal to $1+\epsilon$ (everyone uses a costless link to node $v$ and then the common link to $t$ ). As $\epsilon$ goes to zero, the price of stability approaches to $H(k)$.

Finally, if there is no equilibrium then the price of stability equals to infinity.

\subsection{Lower bound for $\operatorname{PoS}(\mathrm{PR})$}

Proof. Consider the network as shown in figure 12. We show that the unique equilibrium of the proportional method is of order $k$. Let the costs of links $s_{i} \rightarrow t$ be $x_{i}$. Straightforward computations show that the $k-t h$ agent will deviate from the efficient graph of cost $1+\epsilon$ if $x_{k} \leq \frac{1-k+\sqrt{(k-1)^{2}+4 k(k-1)}}{2 k}$. As $k$ grows, $x_{k}$ converges to the golden number $\frac{\sqrt{5}-1}{2}$ in contrast to $1 / k$ for the uniform method, which goes to zero. Also $x_{t-1}>x_{t}$ for all $t=2,3, . ., k$ and $x_{1}=1$. Thus the lower bound on the PoS of the proportional method is $\sum_{i=1}^{k} x_{i}$, which is of order $k$.

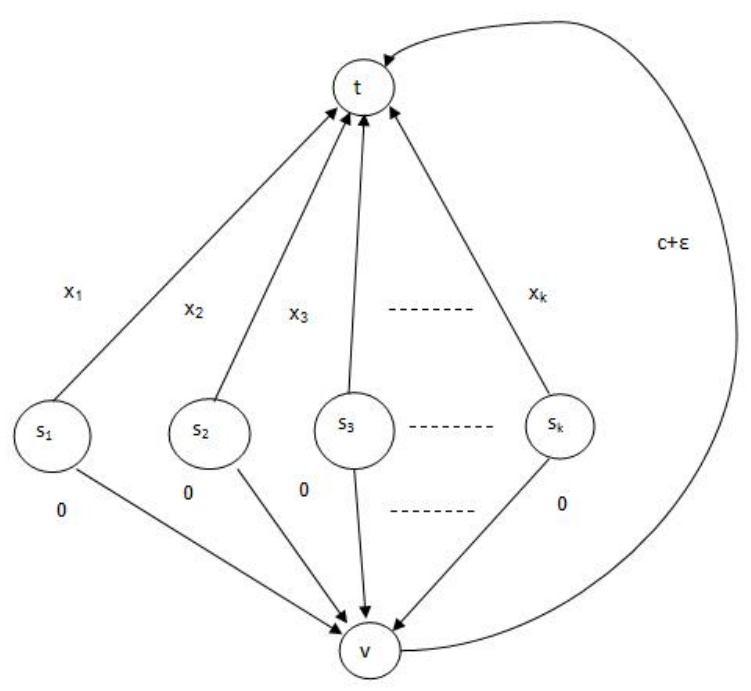

Figure 12: $\operatorname{PoS}(\mathrm{PR})$ is of order $k$.

\section{Other proofs}

\subsection{Proof of Proposition 2}

Proof. 
The proof is very similar to the existence of Nash equilibrium for AEG in lemma 2 and details are omitted to avoid repetition.

By step 1 in section 8.1, there exist an index $m$ and $\lambda$ such that

i. $\varphi_{i}=s^{i}$ for $i=1, \ldots, m$

ii. $\varphi_{h}=f^{h}(\lambda)$ for $h>m$.

iii. $g^{m}\left(s^{m}\right)<\lambda \leq g^{m+1}\left(s^{m+1}\right)$

Assume there are deviations by a group of agent from the equilibrium with the minimal cost $c^{1}$. Then $\lambda$ will decrease at every step. If we continue iterating until there is no deviations, then in a finite number of iterations we converge to a profile $Y$ such that agents either pay a common value $\tilde{\lambda}$ or their stand-alone cost $f^{i}\left(s^{i}\right)$. Since no group of agents can deviate, then $Y$ is a Nash equilibrium of cost smaller than $c^{1}$, which is a contradiction.

\subsection{The game generated by EG does not admit an ordinal potential}

Consider a network shown in figure 13 below. Here there are two agents- agent 1 and agent 2 with their demand nodes being $\left\{s_{1}, t\right\}$ and $\left\{s_{2}, t\right\}$ respectively. Both the agents have two strategies each. One of the strategies of the agent $i$ $(i=1,2)$ is to connect through the direct link, i.e $s_{i} \rightarrow t$ and her other strategy is to connect indirectly through the node $v$, i.e., $s_{i} \rightarrow v \rightarrow t$. Let's denote the two strategies of agent 1 as $a \& b$ and the two strategies of agent 2 as $c \& d$ where $a:=s_{1} \rightarrow v \rightarrow t, b:=s_{1} \rightarrow t, c:=s_{2} \rightarrow t$ and $d:=s_{2} \rightarrow v \rightarrow t$. Given the $E G$ mechanism, the game induced by the network on the set of agents can be represented in normal form by the following matrix, where the agent 1 is the row player and agent 2 is the column player. The first numbers in each cell of the matrix correspond to the cost share (negative of payoff) of agent 1 and the second to that of the agent 2 .

$\begin{array}{ccc} & c & d \\ a & 3,1.5 & 1.5,1.5 \\ b & 2,1.5 & 2,1.5\end{array}$

Suppose, that this is an ordinal potential game. Then, there must exist an ordinal potential function $P:\{a, b\} \times\{c, d\} \rightarrow \mathbb{R}$ satisfying $P(a, c)>P(b, c)=$ $P(b, d)>P(a, d)=P(a, c)$ which is impossible. 


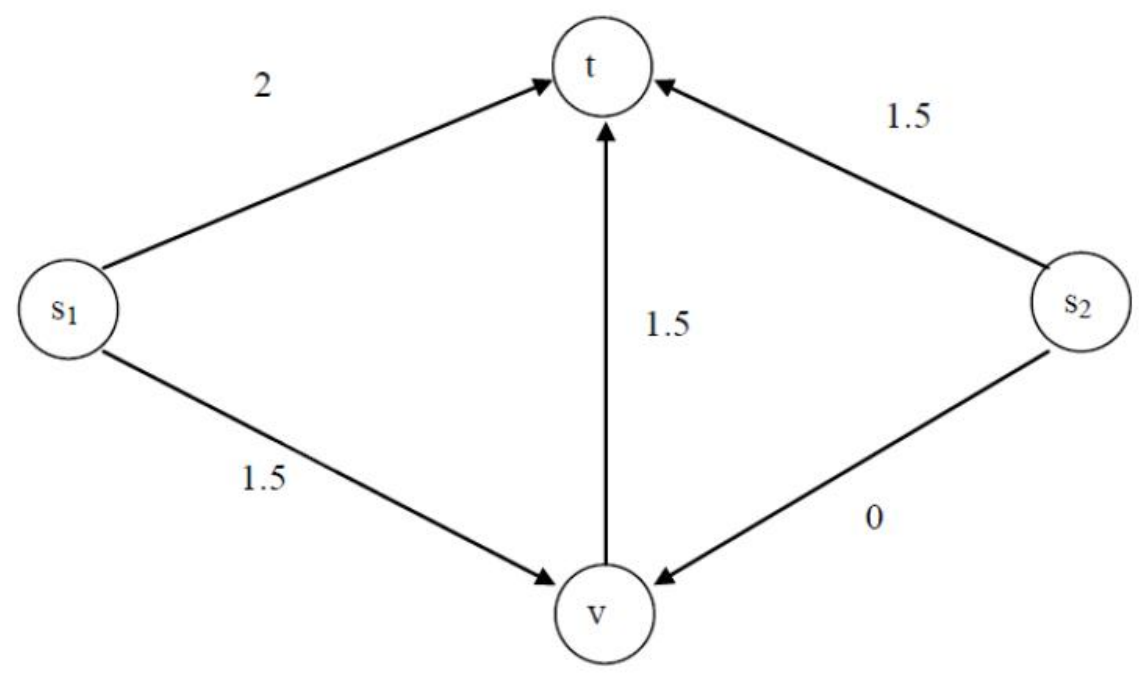

Figure 13: Network illustrating EG does not admit a potential.

\section{References}

[1] Fiat, A., Kaplan, H., Levy, M., Olonetsky, S., and Shabo, R. 2006. On the price of stability for designing undirected networks with fair cost allocations, in: ICALP06.

[2] Andelman, N., Feldman, M., and Mansour, Y., 2009. Strong price of anarchy, Games and Economic Behavior 65, (2009) 289-317

[3] Anshelevich E., Dasgupta A., Kleinberg L., Tardos E., Wexler T., and Roughgarden T., 2004. The Price of Stability for Network Design with Fair Cost Allocation. In 45th Annual IEEE Symposium on Foundations of Computer Science (FOCS), pages 59-73, 2004.

[4] Chen, H. L., Roughgarden, T., Valiant, G., 2008. Designing Networks with good Equilibria, Proceedings of the 19th annual ACM-SIAM Symposium on Discrete Algorithms.

[5] Epstein, A., Feldman, M. and Mansour, Y., 2007. Strong Equilibrium in cost sharing connection games, ACM conference on Electronic Commerce, San Diego.

[6] Epstein, A., Feldman, M. and Mansour, Y., 2009. Strong Equilibrium in cost sharing connection games, Games and Economic Behavior 67, (2009) $51-68$. 
[7] Green, J., and Laffont, J. 1979. Incentives in public decision-making. NorthHolland Publishing Company.

[8] Hougaard, J. L. and Tvede, M. 2010. Truth-Telling and Nash Equilibria in Minimum Cost Spanning Tree Models. Working Paper, Department of food and resource Economics, University of Copenhagen.

[9] http://www.foxnews.com/story/0,2933,475507,00.html

[10] http://www.msnbc.msn.com/id/28472161/

[11] http://archives.chicagotribune.com/2009/jan/05/nation/chi-oregongasjan05

[12] http://watchingthewatchers.org/indepth/24150/gas-vs-mileage-tax

[13] http://www.kansascity.com/105/story/1299981.html

[14] http://www.miamiherald.com/news/florida/AP/story/1281003.html

[15] Juarez, R. 2008. Group strategyproof cost sharing: the role of indifferences. Under Review.

[16] Juarez, R. 2008. The worst absolute surplus loss in the problem of commons: Random Priority vs. Average Cost. Economic Theory 34.

[17] E. Koutsoupias, C. H. Papadimitriou, 1999. Worst-case equilibria, STACS 99.

[18] Kumar, R. 2009. Inefficiency of Shapley Mechanism and some alternatives. Mimeo Rice University.

[19] Jian Li., 2009. An upper bound on the price of stability for undirected Shapley network design games. Information Processing Letters 2009.

[20] Marden, J. R., Wierman, A., 2008. Distributed Welfare Games, Forthcoming in Operations Research.

[21] Maskin, E., T Sjostrom. In K. J. Arrow, A. K. Sen, K. Suzumura (ed.), Handbook of Social Choice and Welfare, 237-282.

[22] Monderer, D., and L. Shapley 1996. Fictitious play property for games with identical interests, Journal of Economic Theory 68, 258-65.

[23] Monderer, D, and L. Shapley, 1996. Potential Games, Games and Economic Behavior, 14, 124-43.

[24] Moulin, H. Incremental Cost Sharing: characterization by coalitional strategy-proofness, Soc. Choice Welfare 16 (1999), 279-320.

[25] Moulin, H., 2008. The price of anarchy of serial, average and incremental cost sharing. Economic Theory, 36, 379-405. 
[26] Moulin, H., 2009. Pricing traffic in spanning network. Proceedings of the ACM conference on Electronic Commerce, Stanford.

[27] Moulin, H. and Shenker, S. 2001. Strategyproof Sharing of Submodular Costs: Budget Balance versus Efficiency, Economic Theory, 18, 3, 511-533.

[28] Moulin, H., 2002. Axiomatic cost and surplus sharing. In K. J. Arrow, A. K. Sen, K. Suzumura (ed.), Handbook of Social Choice and Welfare, 289-357.

[29] Sharkey, W. W., Network models in economics, in: Ball, M. O., Magnanti, T. L., Nonma, C. L., Nemhauser, G. L. (Eds.), Handbooks in Operation Research and Management Science, Elsevier, New York, 1995, pp. 713-765.

[30] Saijo, T., Sjöström, T., Yamato, T., (2007). Secure implementation. Theoretical Economics 2, 203-229.

[31] Sandholm, W., 2001. Potential games with continuous player sets, Journal of Economic Theory, 97, 81-108.

[32] Sprumont, Y. 1991. The division problem with single-peaked preferences: A characterization of the uniform allocation rule. Econometrica, 59, 2: 509519.

[33] Thomson, W. 2003. Axiomatic and game-theoretic analysis of bankruptcy and taxation problems: a survey. Mathematical Social Sciences 45 249-297.

[34] Young, H. P. 1987. On dividing an amount according to individual claims or liabilities. Mathematics of Operations Research, 12: 398-414. 